Research Article

\title{
Effective Negative Mass Nonlinear Acoustic Metamaterial with Pure Cubic Oscillator
}

\author{
Ming Gao $\mathbb{D}^{1,2}$ Zhiqiang Wu ${ }^{10},{ }^{1}$ and Zhijie Wen ${ }^{2}{ }^{2}$ \\ ${ }^{1}$ Department of Mechanics, School of Mechanical Engineering, Tianjin University, Tianjin, China \\ ${ }^{2}$ College of Mining and Safety Engineering, Shandong University of Science and Technology, Qingdao, China
}

Correspondence should be addressed to Zhiqiang Wu; zhiqwu@tju.edu.cn and Zhijie Wen; sdust0532@gmail.com

Received 4 May 2018; Accepted 11 July 2018; Published 30 September 2018

Academic Editor: Fengqiang Gong

Copyright (c) 2018 Ming Gao et al. This is an open access article distributed under the Creative Commons Attribution License, which permits unrestricted use, distribution, and reproduction in any medium, provided the original work is properly cited.

Acoustic metamaterial, which can prohibit effectively the elastic wave propagation in the bandgap frequency range, has broad application prospects in the vibration and noise reduction areas. The Lindstedt-Poincaré method was utilized to analyze the dispersion curves of nonlinear metamaterial with a pure Duffing oscillator. The first-order perturbation solutions of acoustic and optical branches were obtained. Both the starting and cutoff frequencies of the bandgap are determined consequently. It was found that the soft/hard characteristics of pure Duffing oscillators could lead to the lower/upper movement of the starting and cutoff frequencies of the bandgap. By further researching the degraded linear system, the conclusion that actual nonlinear metamaterial bandgap region is wider than effective negative mass region is drawn and that both mass and stiffness ratio effect on the starting frequency is obtained. Effective positive mass can also lead to the vibration attenuation in bandgap. For nonlinear metamaterial, the translation effect of the external excitation amplitude on the bandgap range and the zero mass at the nonlinear bandgap cutoff frequency were discussed, and all above conclusion are identified by numerical analysis.

\section{Introduction}

In the last ten years, a significant attention is directed toward so called metamaterial, a kind of artificial structures, which is composed of small substructure that behaves like a continuous material. The most remarkable property of metamaterials is the bandgap in which the acoustic/elastic waves propagate is prohibited [1]. With this unique characteristic, metamaterials can be applied to the wave filtering [2-4], vibration attenuation [5], acoustic isolation [6, 7], and sonic transmission $[8,9]$. In civil engineering, acoustic metamaterials with the band characteristics were also created to replace the traditional seismic designs for isolating seismic waves from buildings. Shi and his coworkers [10-14] proposed a novel concept of foundation, periodic foundation, to isolate civil structures from seismic wave with frequencies that might resonate with the structures, which has been investigated in a number of comprehensive theoretical, numerical, and experimental studies. In particular, Brûlé et al. [15] carried out the large-scale test of seismic metamaterials [15] such as seismic metawedge capable [16] capable of creating seismic waves and studied the attenuation effect of seismic waves by using finite periodic lattices of mass-in-mass barriers [17].

Effective negative mass metamaterials come from mass effective ratio, an artificial parameter, which is calculated analogically as the parameter of electromagnetic metamaterials $[18,19]$ and has not only positive but also negative values. Based on the idea of locally resonant microstructure, Liu et al. [20] designed the first effective negative mass metamaterial in certain frequency region. Subsequently, Liu et al. [21], Milton and Willis [22], Willis [23], and Huang et al. [24, 25] proposed various analytic models to analyze the related effective negative mass mechanisms. Yao et al. [26] realized effective negative mass and zero-mass phenomena in a $1 \mathrm{D}$ mass-spring system experimentally. Other different types of acoustic metamaterials with negative effective mass have been proposed [27-32]. From the existing literatures, the starting frequency bandgap of metamaterials is defined by local resonance frequency where the effective negative mass just began. However, the bandgap starting frequency will change with outer spring stiffness when the local resonance frequency 
remains unchanged [26], and actually the starting frequency is lower than resonance frequency in experimental research.

Nonlinear acoustic metamaterials also deserve special attention. Vakakis and his coauthors [33, 34] utilized the multiple scales perturbation method to analyze nonlinear chains subjected to external forcing and ground springs and developed nonlinear dispersion relationships that exhibit amplitude dependence. Chakraborty and Mallik [35] studied the cubic chain and the effect of nonlinearities on the propagation constant and natural frequencies. Then Lazarov and Jensen [36] considered a linear chain with attached nonlinear damped oscillators by balance approach. Marathe and Chatterjee [37] looked at a damped nonlinearity and used harmonic balance and multiple scales to uncover the decay rate in the propagation zone. Narisetti et al. [38] developed a Lindstedt-Poincaré perturbation technique and analyzed monoatomic cubic chains to capture dispersion and bandgap shifts. However, their models are not based on effective negative mass, and Duffing oscillator is often a damped one. The damp may have great influence on the starting and cutoff frequencies of bandgap [39]. As a result, in order to investigate how effect of the nonlinearity on the effective negative mass nonlinear metamaterial dispersion curves, the pure Duffing oscillator, which is no damped pure Duffing oscillator is adaptive

It is worth mentioning that, there is no literature discussion whether positive effective mass phenomenon exists in the nonlinear metamaterial bandgap based on effective negative mass.

In this paper, effective negative mass nonlinear metamaterial mathematical model is given firstly, and Lindstedt-Poincaré perturbation method is utilized to calculate metamaterial dispersion curves. Then, the precise expression of both starting and cutoff frequencies is deduced consequently. After that, the result is obtained that positive effective mass phenomenon exists in the nonlinear bandgap. Finally, the numerical calculation verification results agree with our theoretical analysis.

\section{The First-Order Solution for the Dispersive Curve of Acoustic Metamaterial of Effective Negative Mass with Pure Cubic Oscillator}

Consider an acoustic metamaterial containing a pure Duffing oscillator, illustrated as Figure 1. The shell mass is $M$, the rigidity coefficient of the spring connecting the two shells is $K$, and the mass of the internal oscillator is $m$. The internal oscillator is a pure Duffing oscillator whose force is proportional to displacement and the cubic of displacement, and the linear rigidity coefficient of the spring connecting the oscillator and the shell is $k$; the coefficient of the cubic term is $\Gamma$.

In the case of period $j$, for instance, $u_{j, 1}$ and $u_{j, 2}$ are the displacements of the shell and the oscillator in period $j ; u_{j-1,1}$ and $u_{j-1,2}$ are the displacements of the shell and the oscillator in period $j-1$; and $u_{j+1,1}$ and $u_{j+1,2}$ are the displacements of the shell and the oscillator in period $j+1$. Next, we are going to discuss kinematic modeling of this system.

From the load on the shell in period $j$, its differential equation of motion is

$$
\begin{aligned}
& M \ddot{u}_{j, 1}+K\left(2 u_{j, 1}-u_{j-1,1}-u_{j+1,1}\right)-2 k\left(u_{j, 2}-u_{j, 1}\right) \\
& -2 \varepsilon \Gamma\left(u_{j, 2}-u_{j, 1}\right)^{3}=0,
\end{aligned}
$$

where overdots denote derivative with respect to time $t$.

According to the load on the oscillator in period $j$, its differential equation of motion is

$$
m \ddot{u}_{j, 2}=-2 k\left(u_{j, 2}-u_{j, 1}\right)-2 \varepsilon \Gamma\left(u_{j, 2}-u_{j, 1}\right)^{3} .
$$

Rewrite Equations (1) and (2) into a matrix

$$
\begin{gathered}
{\left[\begin{array}{cc}
M & 0 \\
0 & m
\end{array}\right]\left[\begin{array}{l}
\ddot{u}_{j, 1} \\
\ddot{u}_{j, 2}
\end{array}\right]+\left[\begin{array}{cc}
2(K+k) & -2 k \\
-2 k & 2 k
\end{array}\right]\left[\begin{array}{l}
u_{j, 1} \\
u_{j, 2}
\end{array}\right]} \\
-\left[\begin{array}{c}
K\left(u_{j-1,1}+u_{j+1,1}\right) \\
0
\end{array}\right]+2 \varepsilon \Gamma\left[\begin{array}{c}
-\left(u_{j, 2}-u_{j, 1}\right)^{3} \\
\left(u_{j, 2}-u_{j, 1}\right)^{3}
\end{array}\right]=0 .
\end{gathered}
$$

Define the external/internal spring rigidity ratio as $\alpha=K / k$, the shell/oscillator mass ratio as $\beta=M / m$, and the linear natural frequency of oscillator as $\omega_{n}=\sqrt{2 k / m}$ by introducing dimensionless time $\tau=\omega t$ and dimensionless nonlinear coefficient $\bar{\Gamma}=\Gamma / m \omega_{n}^{2}$. This way, we can nondimensionalize Equation (3):

$$
\begin{gathered}
\bar{\omega}^{2}\left[\begin{array}{ll}
\beta & 0 \\
0 & 1
\end{array}\right]\left[\begin{array}{c}
\frac{d^{2} u_{j, 1}}{d \tau^{2}} \\
\frac{d^{2} u_{j, 2}}{d \tau^{2}}
\end{array}\right]+\left[\begin{array}{cc}
1+\alpha & -1 \\
-1 & 1
\end{array}\right]\left[\begin{array}{l}
u_{j, 1} \\
u_{j, 2}
\end{array}\right] \\
-\frac{\alpha}{2}\left[\begin{array}{c}
u_{j-1,1}+u_{j+1,1} \\
0
\end{array}\right]+2 \varepsilon \bar{\Gamma}\left[\begin{array}{c}
-\left(u_{j, 2}-u_{j, 1}\right)^{3} \\
\left(u_{j, 2}-u_{j, 1}\right)^{3}
\end{array}\right]=0,
\end{gathered}
$$

here $\bar{\omega}=\omega / \omega_{n}$ is a dimensionless frequency. Now we are going to find the first-order perturbation solution of $\bar{\omega}$ by Lindstedt-Poincaré method.

Using the asymptotic expansion below:

$$
\left\{\begin{array}{l}
u_{j}=u_{j}^{(0)}+\varepsilon u_{j}^{(1)}+o\left(\varepsilon^{2}\right), \\
\bar{\omega}=\bar{\omega}_{0}+\varepsilon \bar{\omega}_{0}+o\left(\varepsilon^{2}\right),
\end{array}\right.
$$

where the superscript and subscript 0 and 1 are the linear and first-order asymptotic expansion alternatively.

Substitute Equation (5) into Equation (4):

$$
\begin{aligned}
& \left(\bar{\omega}_{0}+\varepsilon \bar{\omega}_{1}\right)^{2}\left[\begin{array}{ll}
\beta & 0 \\
0 & 1
\end{array}\right]\left[\begin{array}{l}
\frac{d^{2} u_{j, 1}^{(0)}}{d \tau^{2}}+\varepsilon \frac{d^{2} u_{j, 1}^{(1)}}{d \tau^{2}} \\
\frac{d^{2} u_{j, 2}^{(0)}}{d \tau^{2}}+\varepsilon \frac{d^{2} u_{j, 2}^{(1)}}{d \tau^{2}}
\end{array}\right]+\left[\begin{array}{cc}
1+\alpha & -1 \\
-1 & 1
\end{array}\right] \\
& .\left[\begin{array}{c}
u_{j, 1}^{(0)}+\varepsilon u_{j, 1}^{(1)} \\
u_{j, 2}^{(0)}+\varepsilon u_{j, 2}^{(1)}
\end{array}\right]-\frac{\alpha}{2}\left[\begin{array}{c}
u_{j-1,1}^{(0)}+u_{j+1,1}^{(0)}+\varepsilon\left(u_{j-1,1}^{(1)}+u_{j+1,1}^{(1)}\right) \\
0
\end{array}\right] \\
& +2 \varepsilon \bar{\Gamma}\left[\begin{array}{c}
-\left(u_{j, 2}^{(0)}-u_{j, 1}^{(0)}\right)^{3} \\
\left(u_{j, 2}^{(0)}-u_{j, 1}^{(0)}\right)^{3}
\end{array}\right] o\left(\varepsilon^{2}\right)=0 .
\end{aligned}
$$




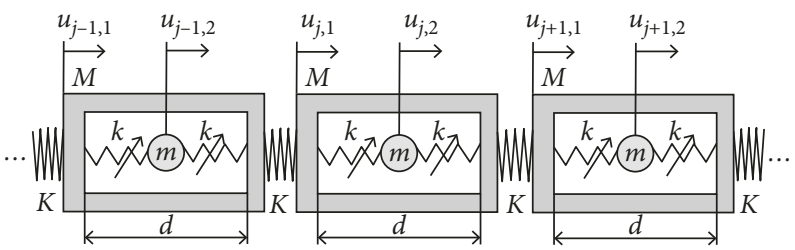

Figure 1: Metamaterial effective negative mass with the pure Duffing oscillator.

Separate the $\varepsilon^{0}$ and $\varepsilon^{1}$ coefficients and order them to be zero. Then, we get,

$\varepsilon^{0}$ coefficient:

$$
\begin{gathered}
\bar{\omega}_{0}^{2}\left[\begin{array}{ll}
\beta & 0 \\
0 & 1
\end{array}\right]\left[\begin{array}{c}
\frac{d^{2} u_{j, 1}^{(0)}}{d \tau^{2}} \\
\frac{d^{2} u_{j, 2}^{(0)}}{d \tau^{2}}
\end{array}\right]+\left[\begin{array}{cc}
1+\alpha & -1 \\
-1 & 1
\end{array}\right]\left[\begin{array}{c}
u_{j, 1}^{(0)} \\
u_{j, 2}^{(0)}
\end{array}\right] \\
-\frac{\alpha}{2}\left[\begin{array}{c}
u_{j-1,1}^{(0)}+u_{j+1,1}^{(0)} \\
0
\end{array}\right]=0 .
\end{gathered}
$$

$\mathcal{E}^{1}$ coefficient:

$$
\begin{gathered}
\bar{\omega}_{0}^{2}\left[\begin{array}{ll}
\beta & 0 \\
0 & 1
\end{array}\right]\left[\begin{array}{c}
\frac{d^{2} u_{j, 1}^{(1)}}{d \tau^{2}} \\
\frac{d^{2} u_{j, 2}^{(1)}}{d \tau^{2}}
\end{array}\right]+\left[\begin{array}{cc}
1+\alpha & -1 \\
-1 & 1
\end{array}\right]\left[\begin{array}{l}
u_{j, 1}^{(1)} \\
u_{j, 2}^{(1)}
\end{array}\right]-\frac{\alpha}{2}\left[\begin{array}{c}
u_{j-1,1}^{(1)}+u_{j+1,1}^{(1)} \\
0
\end{array}\right] \\
=-2 \bar{\omega}_{0} \bar{\omega}_{1}\left[\begin{array}{ll}
\beta & 0 \\
0 & 1
\end{array}\right]\left[\begin{array}{l}
\frac{d^{2} u_{j, 1}^{(0)}}{d \tau^{2}} \\
\frac{d^{2} u_{j, 2}^{(0)}}{d \tau^{2}}
\end{array}\right]-2 \bar{\Gamma}\left[\begin{array}{c}
-\left(u_{j, 2}^{(0)}-u_{j, 1}^{(0)}\right)^{3} \\
\left(u_{j, 2}^{(0)}-u_{j, 1}^{(0)}\right)^{3}
\end{array}\right] .
\end{gathered}
$$

2.1. Linear Dispersive Curve Equation. The steady-state solution of Equation (7) is expressed as

$$
\begin{gathered}
{\left[\begin{array}{l}
u_{j, 1}^{(0)} \\
u_{j, 2}^{(0)}
\end{array}\right]=\left[\begin{array}{c}
A_{1}^{(0)} \\
A_{2}^{(0)}
\end{array}\right] \frac{1}{2} e^{i q a j} e^{i \tau}+\text { c.c., }} \\
{\left[\begin{array}{l}
u_{j \pm 1,1}^{(0)} \\
u_{j \pm 1,2}^{(0)}
\end{array}\right]=\left[\begin{array}{l}
A_{1}^{(0)} \\
A_{2}^{(0)}
\end{array}\right] \frac{1}{2} e^{i q a(j \pm 1)} e^{i \tau}+\text { c.c. },}
\end{gathered}
$$

where $a$ is the periodic unit size, qaj is the $j$ periodic phase factor, and $A_{1}^{(0)} A_{2}^{(0)}$ are the steady-state amplitude of shell and oscillator, respectively. c.c. stands for the conjugation of the equations above.

Substitute Equations (9) and (10) into Equation (7):

$$
\left[\begin{array}{cc}
-\bar{\omega}_{0}^{2} \beta+1+2 \alpha \sin ^{2}\left(\frac{q a}{2}\right) & -1 \\
-1 & 1-\bar{\omega}_{0}^{2}
\end{array}\right]\left[\begin{array}{c}
A_{1}^{(0)} \\
A_{2}^{(0)}
\end{array}\right] \frac{1}{2} e^{i q a j} e^{i \tau}+\text { c.c. }=0 .
$$

In order for Equation (11) to have nonzero solutions $A_{1}^{(0)} A_{2}^{(0)}$, the determinant of its coefficient matrix must be zero. Hence,

$$
\bar{\omega}_{0}^{2}\left(\beta+\frac{1}{1-\bar{\omega}_{0}^{2}}\right)=2 \alpha \sin ^{2}\left(\frac{q a}{2}\right),
$$

and $A_{1}^{(0)}$ and $A_{2}^{(0)}$ have the following relation:

$$
A_{1}^{(0)}=\left(1-\bar{\omega}_{0}^{2}\right) A_{2}^{(0)} \text {. }
$$

By finding $\bar{\omega}_{0}^{2}$ in Equation (12), we get the dispersive curve equation of the two frequency branches:

$$
\begin{aligned}
& \bar{\omega}_{0}^{\mathrm{aco}}=\frac{\sqrt{2} \sqrt{\beta\left[\beta+1+2 \alpha \sin ^{2}(q a / 2)-\sqrt{(\beta+1)^{2}+4 \alpha \sin ^{2}(q a / 2)\left(\alpha \sin ^{2}(q a / 2)-\beta+1\right)}\right]}}{2 \beta}, \\
& \bar{\omega}_{0}^{\mathrm{opt}}=\frac{\sqrt{2} \sqrt{\beta\left[\beta+1+2 \alpha \sin ^{2}(q a / 2)+\sqrt{(\beta+1)^{2}+4 \alpha \sin ^{2}(q a / 2)\left(\alpha \sin ^{2}(q a / 2)-\beta+1\right)}\right]}}{2 \beta},
\end{aligned}
$$

where $\bar{\omega}_{0}^{\mathrm{aco}}$ and $\bar{\omega}_{0}^{\mathrm{opt}}$ stand for the acoustic and optical frequency branches. An acoustic frequency branch is a lowfrequency dispersive curve while an optical frequency branch is a high-frequency dispersive curve. On this basis, we can obtain linear dispersive curve Equations (14) and (15). 
2.2. Nonlinear Dispersive Curve Equation. Next, let us consider the first-order perturbation solution which, for Equation (8), is expressed as

$$
\begin{gathered}
{\left[\begin{array}{c}
u_{j, 1}^{(1)} \\
u_{j, 2}^{(1)}
\end{array}\right]=\left[\begin{array}{c}
A_{1}^{(1)} \\
A_{2}^{(1)}
\end{array}\right] \frac{1}{2} e^{i q a j} e^{i \tau}+\text { c.c., }} \\
{\left[\begin{array}{c}
u_{j \pm 1,1}^{(1)} \\
u_{j \pm 1,2}^{(1)}
\end{array}\right]=\left[\begin{array}{c}
A_{1}^{(1)} \\
A_{2}^{(1)}
\end{array}\right] \frac{1}{2} e^{i q a(j \pm 1)} e^{i \tau}+\text { c.c. }}
\end{gathered}
$$

Substitute Equations (16) and (17) into Equation (8):

$$
\begin{aligned}
& {\left[\begin{array}{cc}
-\bar{\omega}_{0}^{2} \beta+1+2 \alpha \sin ^{2}\left(\frac{q a}{2}\right) & -1 \\
-1 & 1-\bar{\omega}_{0}^{2}
\end{array}\right]\left[\begin{array}{c}
A_{1}^{(1)} \\
A_{2}^{(1)}
\end{array}\right] \frac{1}{2} e^{i q a j} e^{i \tau}+\text { c.c. }} \\
& =\left[\begin{array}{c}
-2 \bar{\omega}_{0} \bar{\omega}_{1} A_{1}^{(0)}+4 \bar{\Gamma} N^{(0)} \\
-2 \bar{\omega}_{0} \bar{\omega}_{1} A_{2}^{(0)}-4 \bar{\Gamma} N^{(0)}
\end{array}\right] \frac{1}{2} e^{i q a j} e^{i \tau} \\
& +\left[\begin{array}{c}
A_{1}^{(0)} \\
A_{2}^{(0)}
\end{array}\right] C e^{3 i q a j} e^{3 i \tau}+\text { c.c. }
\end{aligned}
$$

where $N^{(0)}=(3 / 4)\left(\bar{A}_{2}^{(0)}-\bar{A}_{1}^{(0)}\right)\left(A_{2}^{(0)}-A_{1}^{(0)}\right)^{2}$.

To remove the secular term, considering only the equality between the $e^{i q a j} e^{i \tau}$ coefficients on both sides of Equation (18). Then we have

$$
\begin{gathered}
{\left[\begin{array}{cc}
-\bar{\omega}_{0}^{2} \beta+1+2 \alpha \sin ^{2}\left(\frac{q a}{2}\right) & -1 \\
-1 & 1-\bar{\omega}_{0}^{2}
\end{array}\right]\left[\begin{array}{l}
A_{1}^{(1)} \\
A_{2}^{(1)}
\end{array}\right]} \\
=\left[\begin{array}{c}
-2 \bar{\omega}_{0} \bar{\omega}_{1} A_{1}^{(0)}+4 \bar{\Gamma} N^{(0)} \\
-2 \bar{\omega}_{0} \bar{\omega}_{1} A_{2}^{(0)}-4 \bar{\Gamma} N^{(0)}
\end{array}\right] .
\end{gathered}
$$

Order $C_{1}=-\bar{\omega}_{0}^{2} \beta+1+2 \alpha \sin ^{2}(q a / 2)$, and consider Equation (12). Then, we have

$$
C_{1}=\frac{1}{1-\bar{\omega}_{0}^{2}}
$$

Through linear transform of the augmented matrix of Equation (19), we get

$$
\left(\begin{array}{ccc}
C_{1} & -1 & 2 \bar{\omega}_{0} \bar{\omega}_{1} A_{1}^{(0)}+4 \bar{\Gamma} N^{(0)} \\
0 & 0 & \left(2 \bar{\omega}_{0} \bar{\omega}_{1} A_{2}^{(0)}-4 \bar{\Gamma} N^{(0)}\right) C_{1}+2 \bar{\omega}_{0} \bar{\omega}_{1} \beta A_{1}^{(0)}+4 \bar{\Gamma} N^{(0)}
\end{array}\right)
$$

In order for Equation (19) to have nonzero solutions $A_{1}^{(1)} A_{2}^{(1)}$, the rank of its coefficient matrix must be the same as that of its augmented rank. Hence,

$$
\left(2 \bar{\omega}_{0} \bar{\omega}_{1} A_{2}^{(0)}-4 \bar{\Gamma} N^{(0)}\right) C_{1}+2 \bar{\omega}_{0} \bar{\omega}_{1} \beta A_{1}^{(0)}+4 \bar{\Gamma} N^{(0)} .
$$

We get

$$
\bar{\omega}_{1}=\frac{2 \bar{\Gamma} N^{(0)} \bar{\omega}_{0}}{A_{2}^{(0)}+A_{1}^{(0)} \beta\left(1-\bar{\omega}_{0}^{2}\right)} .
$$

So, the perturbed solution is expressed as

$$
\bar{\omega}=\bar{\omega}_{0}+\varepsilon \bar{\omega}_{1}=\bar{\omega}_{0}+\varepsilon \frac{2 \bar{\Gamma} N^{(0)} \bar{\omega}_{0}}{A_{2}^{(0)}+A_{1}^{(0)} \beta\left(1-\bar{\omega}_{0}^{2}\right)}+o\left(\varepsilon^{2}\right) .
$$

Considering the relation between the $A_{1}^{(0)}$ and $A_{2}^{(0)}$ shown in Equation (13), Equation (23) can be written as

$$
\bar{\omega}=\bar{\omega}_{0}+\varepsilon \frac{3 \bar{\Gamma} \bar{\omega}_{0} 7\left|A_{2}^{(0)}\right|^{2}}{2\left[1+\beta\left(1-\bar{\omega}_{0}^{2}\right)^{2}\right]}+o\left(\varepsilon^{2}\right)
$$

By substituting $\bar{\omega}_{0}^{\text {opt }}$ and $\bar{\omega}_{0}^{\text {aco }}$ shown in Equations (14) and (15) into the equation above, we can obtain the dispersive curve equations of the first-order asymptotically expanded acoustic and optical frequency branches:

$$
\begin{aligned}
& \bar{\omega}_{\mathrm{opt}}=\bar{\omega}_{0}^{\mathrm{opt}}+\varepsilon \frac{3 \bar{\Gamma}\left(\bar{\omega}_{0}^{\mathrm{opt}}\right)^{7}\left|A_{2}^{(0)}\right|^{2}}{2\left\{1+\beta\left[1-\left(\bar{\omega}_{0}^{\mathrm{opt}}\right)^{2}\right]^{2}\right\}}, \\
& \bar{\omega}_{\mathrm{aco}}=\bar{\omega}_{0}^{\mathrm{aco}}+\varepsilon \frac{3 \bar{\Gamma}\left(\bar{\omega}_{0}^{\mathrm{aco}}\right)^{7}\left|A_{2}^{(0)}\right|^{2}}{2\left\{1+\beta\left[1-\left(\bar{\omega}_{0}^{\mathrm{aco}}\right)^{2}\right]^{2}\right\}},
\end{aligned}
$$

These are the dispersive curve equations of the acoustic and optical frequency branches that include for nonlinear factors. We can see that these dispersive curve equations are relevant to nonlinear small perturbation $\varepsilon$, coefficient before nonlinear term $\bar{\Gamma}$, and steadystate vibration amplitude of the pure Duffing oscillator $\left|A_{2}^{(0)}\right|$.

At $\alpha=5, \beta=3, \varepsilon=0.0135$, the influence of coefficient before the nonlinear term $\Gamma$, on the dispersive curve is analyzed as presented in Figure 2 . Here, $\Gamma=1$ represents a hard Duffing oscillator, $\Gamma=-1$ represents a soft Duffing oscillator, and $\Gamma=0$ represents linearity. From this diagram, when the local resonance oscillator is a hard Duffing oscillator, its two dispersive curves will translate toward the high band relative to the linear oscillator; when the local resonance oscillator is a soft Duffing oscillator, on the contrary, its two dispersive curves will translate toward the low band relative to the linear oscillator. Also, under the same conditions, nonlinear factors make greater differences to the dispersive equation of the optical frequency branch than to that of the acoustic frequency branch.

2.3. Rediscussion on the Starting and Cutoff Frequencies of a Corresponding Linear System. For acoustic frequency branch $\bar{\omega}_{0}^{\mathrm{aco}}$ in Equation (14), when $q a=\pi$, the bandgap starting frequency is 


$$
\begin{aligned}
\bar{\omega}_{01} & =\frac{1}{2 \beta} \sqrt{2 \beta\left(\beta+1+2 \alpha-\sqrt{4 \alpha^{2}-4 \alpha \beta+4 \alpha+\beta^{2}+2 \beta+1}\right)}, \\
& =\sqrt{\frac{1}{2 \beta}\left(\beta+1+2 \alpha-\sqrt{4 \alpha^{2}-4 \alpha \beta+4 \alpha+\beta^{2}+2 \beta+1}\right)}, \\
& =\sqrt{\frac{1}{2 \beta}\left[\beta+1+2 \alpha-\sqrt{(-\beta+1+2 \alpha)^{2}+4 \beta}\right]}, \\
& \left.=\sqrt{\frac{1}{2 \beta}\left[2 \beta+(-\beta+1+2 \alpha)-\sqrt{(-\beta+1+2 \alpha)^{2}}+4 \beta\right.}\right] \\
& =\sqrt{1+\frac{(-\beta+1+2 \alpha)-\sqrt{(-\beta+1+2 \alpha)^{2}+4 \beta}}{2 \beta}},
\end{aligned}
$$

It is easy to prove that, in Equation (27), $-\beta+1+2 \alpha+$ $\sqrt{(-\beta+1+2 \alpha)^{2}+4 \beta}>0$. Hence, the bandgap dimensionless starting frequency is smaller than 1 . Namely, the bandgap starting frequency is smaller than the resonance frequency $\omega_{0}$. Furthermore, $\bar{\omega}_{01}=1$ is the start area of effective negative mass. In the literature [26], $\bar{\omega}_{01}=1$ is used to define the bandgap starting frequency. Comparing with the $\bar{\omega}_{01}$ value, we can see that it is higher than the actual starting frequency. It is relatively conservative for bandgap design, but does not reflect the influence of spring stiffness of the shell.

Figure 3 shows how the starting frequency changes when $\alpha$ and $\beta$ are defined differently. From Figure 3, the variation of the nondimensionalized starting frequency $\bar{\omega}_{01}$ is not only relevant to the shell/oscillator rigidity ratio $\alpha$, but also to their mass ratio $\beta$. Hence it is inappropriate to simply define the bandgap starting frequency as a resonance frequency. Literature [26] offers a good example supporting our observation with resonance frequency of $6.35 \mathrm{~Hz}$ and the starting frequency at $5.8 \mathrm{~Hz}$.

For $\bar{\omega}_{0}^{\text {opt }}$ in Equation (15), when $q a=0$, the bandgap cutoff frequency is

$$
\bar{\omega}_{02}=1+\frac{1}{\sqrt{\beta}} .
$$

From Equation (28), the bandgap cutoff frequency is only relevant to the shell/oscillator mass ratio $\beta$ and decreases when the mass ratio increases. And the cutoff frequency is corresponding to the effective zero mass.

Considering the dispersive curve equations of the firstorder asymptotically expanded acoustic and optical frequency branches shown in Equations (25) and (26), for nonlinear acoustic metamaterial with weak nonlinearity $(\varepsilon$ is small), nonlinear system characteristics depend on the corresponding degraded linear systems. As a result, in linear system the phenomenon that both effective and negative

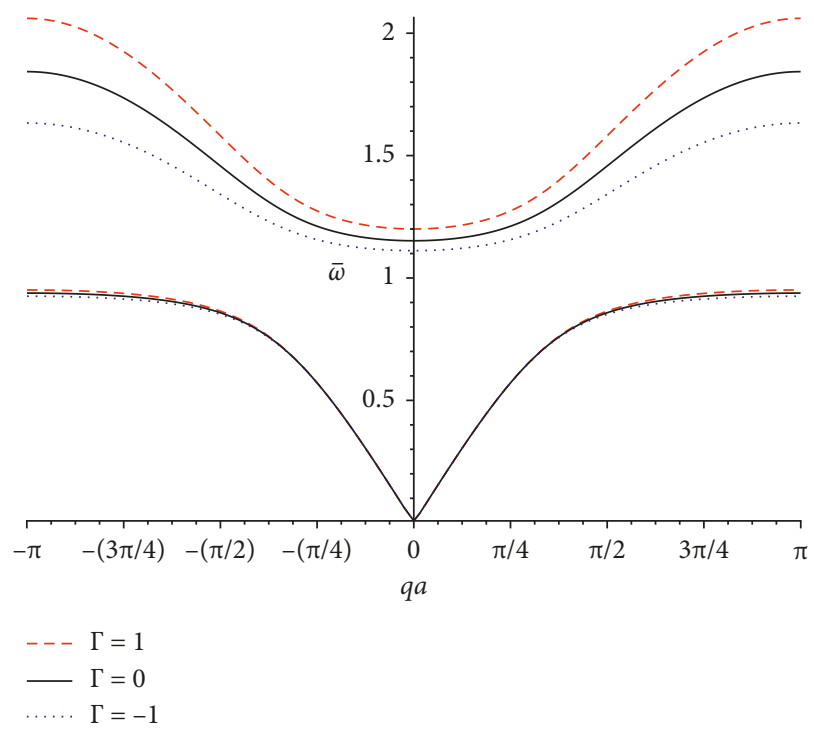

Figure 2: The influence of nonlinear factors on the dispersive curve.

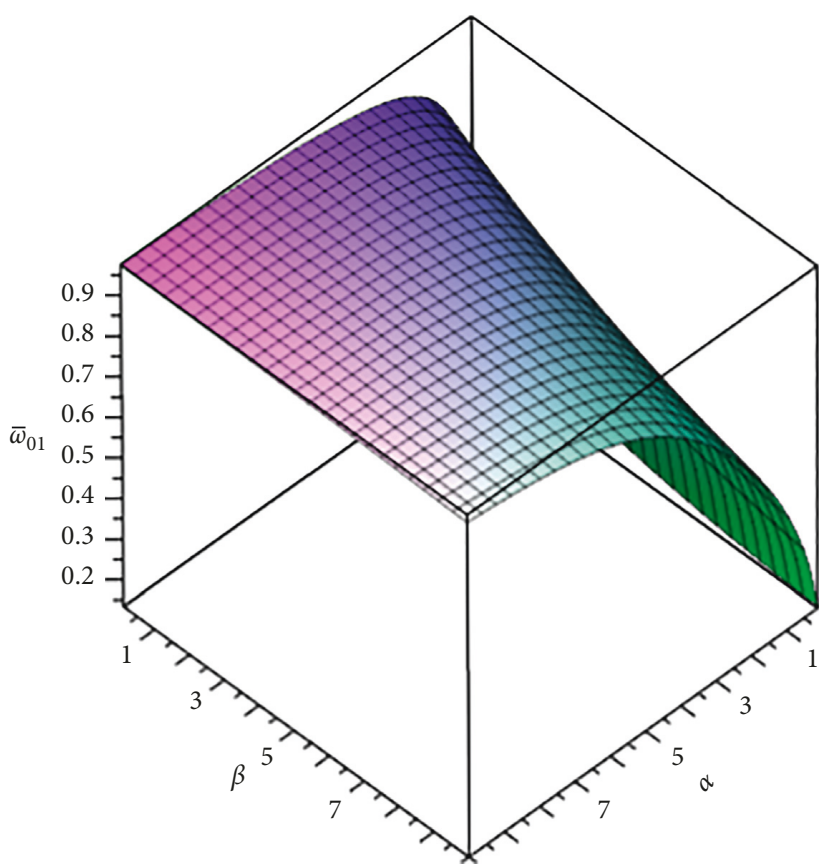

FIgURE 3: The influence of $\alpha$ and $\beta$ on the starting frequency.

mass in bandgap and the phenomenon that cutoff frequency is effective zero mass should also exist in the nonlinear system However, none of the above has been reported. Then, verify them by numerical methods as mentioned in the following.

\section{Numerical Simulation and Validation}

The bandgap characteristics of infinite acoustic metamaterial can be assumed by the finite one (the periodic number is less than 5) [40]. In order to validate our theoretical analysis above, we are going to build a model of an effective negative 


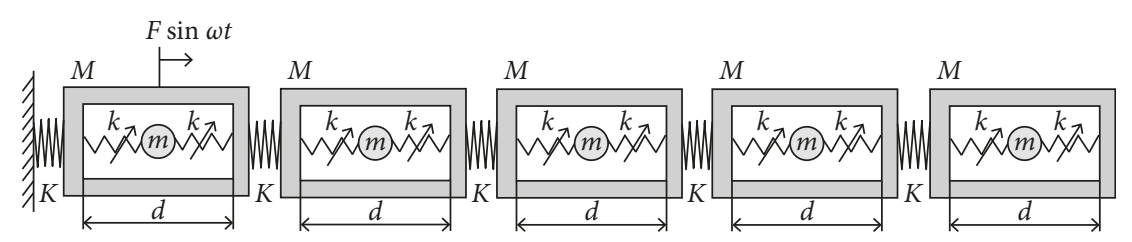

FIGURE 4: Effective negative mass system models of the Duffing oscillator of five different periods.

mass system with pure Duffing oscillator in five periods as shown in Figure 4. By adding a simple harmonic exciting force $F \sin \omega t$ on the shell of period 1 and picking up the response in period 5 , the steady-state displacements of the shell and the oscillator are calculated at each of the excitation frequencies. This way, we get the vibration transmission characteristics of this finite periodic structure to analyze the bandgap and vibration transmission pattern of this structure. When the coefficient before the nonlinear term is $\Gamma=0$, it represents the corresponding linear system.

\subsection{Influence of Nonlinear Terms on the Bandgap Starting and} CutoffFrequencies. To validate the theoretical analysis result above, we may compare it effectively against experimental parameters provided in the literature [26] and establish a periodic structure vibration system of five periods with shell mass $M=0.1011 \mathrm{~kg}$, internal oscillator mass $m=0.04647 \mathrm{~kg}$, internal nonlinear spring linear rigidity $k=37 \mathrm{~N} / \mathrm{m}$, external linear spring damping coefficient $c=0.05 \mathrm{NS} / \mathrm{m}$, and rigidity coefficient of spring linking the external oscillators $K=117 \mathrm{~N} / \mathrm{m}$. If the nonlinear perturbation term is taken as $\varepsilon=0.01$, to facilitate expression, we define

$$
\Gamma=n k
$$

When $n$ is positive, Duffing is hard rigid; when $n$ is negative, Duffing is soft rigid; when $n$ is zero, the system has been degraded into a linear system. The vibration transmission characteristic curves of the corresponding system are calculated at $n=0,1,-1,3,-3,10$, and -10 as presented in Figure 5. The vibration transmission can be confirmed by the ratio of the fifth shell steady-state amplitude to the first shell steady-state amplitude.

As illustrated in Figure 5, existence of a nonlinear term does not make much difference to the bandgap, mainly because the Duffing oscillator we selected is weakly nonlinear. By enlarging the points of the bandgap starting and cutoff frequencies in the chart, we will see how a nonlinearity factor affects the bandgap.

When the longitudinal coordinate (i.e., transmission rate) of the transmission characteristic curve turns from positive to negative, the vibration in period 1 cannot be transmitted to the shell of period 5. Then, a bandgap will appear. Hence, we have to find the point where the transmission curve runs across the horizontal axis, i.e., the bandgap starting frequency, as presented in Figure 6.

From Figure 6(a), when $n$ is positive, the corresponding internal Duffing oscillator is a hardened Duffing oscillator. Hence, we can observe that, as $n$ increases, the bandgap starting frequency gradually translates toward the highfrequency region. When $n$ is negative, the corresponding internal Duffing oscillator is a softened Duffing oscillator. Hence, as $n$ decreases, the bandgap starting frequency gradually translates toward the low-frequency region.

The same method also applies to the influence of nonlinear factor $n$ on the bandgap cutoff frequency. As shown in Figure 6(b), when $n$ is positive, the corresponding internal Duffing oscillator is a hardened Duffing oscillator. Hence, we can observe that, as $\mathrm{n}$ increases, the bandgap cutoff frequency gradually translates toward the high-frequency region. When $n$ is negative, the corresponding internal Duffing oscillator is a softened Duffing oscillator. As $n$ decreases, the bandgap cutoff frequency gradually translates toward the low-frequency region.

As discussed above, existence of nonlinear factor affects the bandgap starting and cutoff frequencies of the structure. The smaller the $n$ value is, the smaller the corresponding starting or cutoff frequency is; the larger the $n$ value is, the larger the starting or cutoff frequency is. Under the same conditions, nonlinear factor makes greater difference to cutoff frequency than it does to starting frequency.

3.2. Influence of the Shell Rigidity on the Bandgap Frequency. As analyzed above, existence of a nonlinear term makes a little difference to the bandgap starting frequency of the corresponding linear system; starting frequency is relevant to shell/oscillator rigidity ratio $\alpha$ and mass ratio $\beta$, and cutoff frequency is only relevant to mass ratio $\beta$. As $\alpha$ decreases, the starting frequency decreases, the cutoff frequency remains the same, and the bandgap width increases. Now, let us draw the vibration transmission profile of the corresponding system by only changing the rigidity coefficient of the spring between the oscillator and the shell, $K$, from $K=117 \mathrm{~N} / \mathrm{m}$ to $37 \mathrm{~N} / \mathrm{m}$ and taking the $n$ value as $n=0,1,-1,3,-3,10$, and -10 as presented in Figure 7.

When the shell connecting rigidity $K=117 \mathrm{~N} / \mathrm{m}$ is changed to $37 \mathrm{~N} / \mathrm{m}$, the masses of the Duffing oscillator and the shell remain the same, and according to analysis results in Equation (27) and Figure 3, after the shell rigidity is changed, the bandgap cutoff frequency will remain the same, but the starting frequency will decrease. That is, the bandwidth of the bandgap will increase, and the bandgap will expand toward the low-frequency region. As can be observed from Figure 5, the bandgap cutoff frequency remains the same at $7.76 \mathrm{~Hz}$ while the bandgap starting frequency has decreased to $3.76 \mathrm{~Hz}$, proving our analysis result. Hence, if we want a wider bandgap width, when other conditions remain unchanged, the rigidity coefficient of the linear 


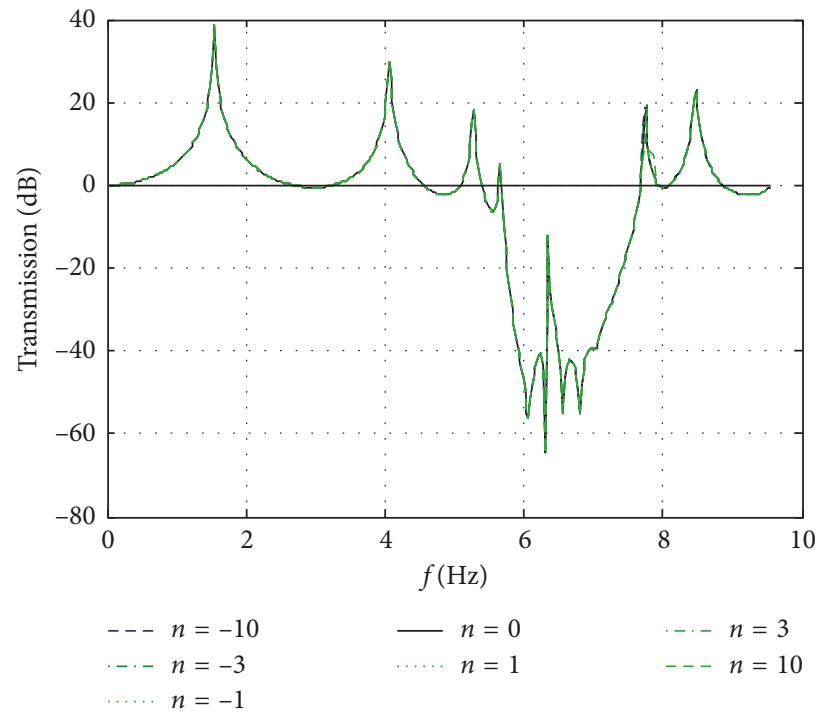

Figure 5: Transmission characteristics with different $n(n=117 \mathrm{~N} / \mathrm{m})$.

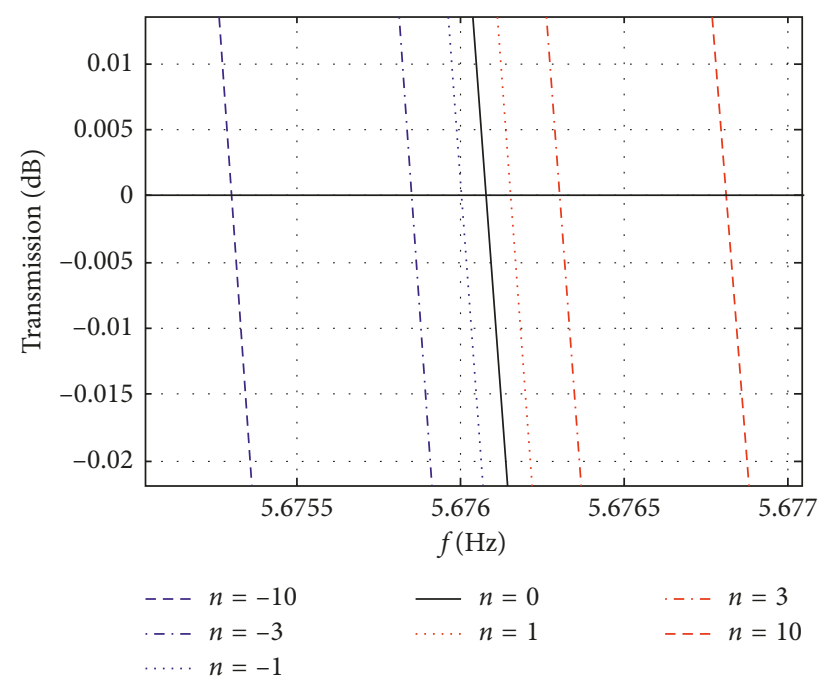

(a)

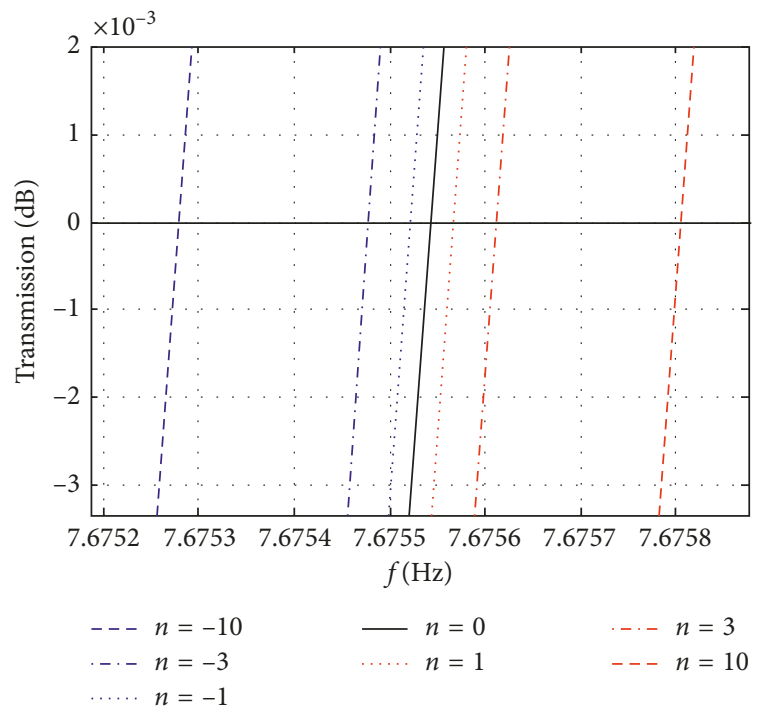

(b)

Figure 6: Starting and cutoff frequencies region $(K=117 \mathrm{~N} / \mathrm{m})$. (a) Starting frequency region. (b) Cutoff frequency region.

spring connecting the shell must be as small as practically possible.

3.3. Responses of the Shell and the Oscillator on the Bandgap. As can be summarized from the discussions on bandgap starting frequency above, existence of weakly nonlinear factor results in some minor changes near the frequency value of its degraded linear system, and that both the Duffing oscillator/shell linear rigidity ratio $\alpha$ and mass ratio $\beta$ make a difference to the starting frequency and that the starting frequency is lower than the resonance frequency of a linear oscillator In other words, generation of bandgap is not always resulted from effective negative mass. Within the spectrum of bandgap, it must be effective positive mass below the linear resonance frequency constituted by the Duffing oscillator and the shell in each period, and effective negative mass does not appear except above the resonance frequency and below the cutoff frequency. Next, the responses of the shells and the oscillators of the system in the effective negative mass area and the effective positive mass area within the spectrum of bandgap are examined with rigidity ratio between springs connecting external large oscillators $K=37 \mathrm{~N} / \mathrm{m}$ while keeping all the other parameters unchanged.

3.3.1. Effective Negative Mass Phenomenon in Bandgap. From Figure 7, with bandgap between $3.7 \mathrm{~Hz}$ and $7.67 \mathrm{~Hz}$ and resonance frequency of linear oscillator at $6.35 \mathrm{~Hz}$, analysis is 


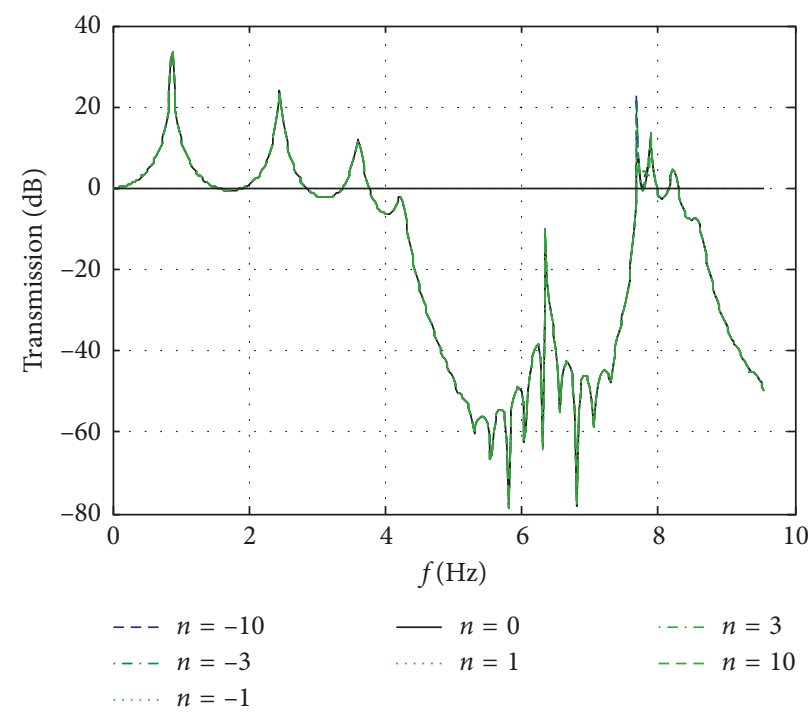

FIgURE 7: Transmission characteristics with different $n(K=37 \mathrm{~N} / \mathrm{m})$.

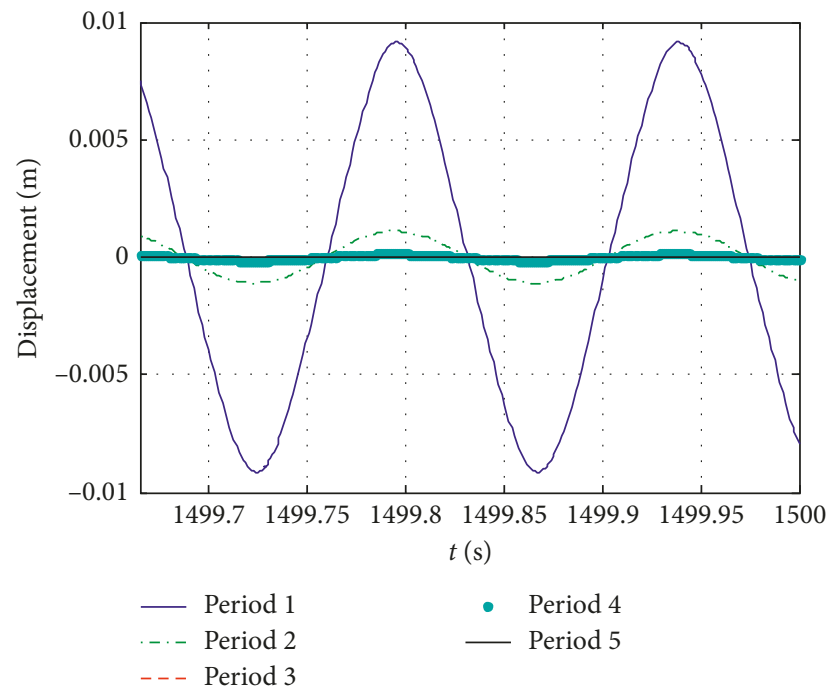

(a)

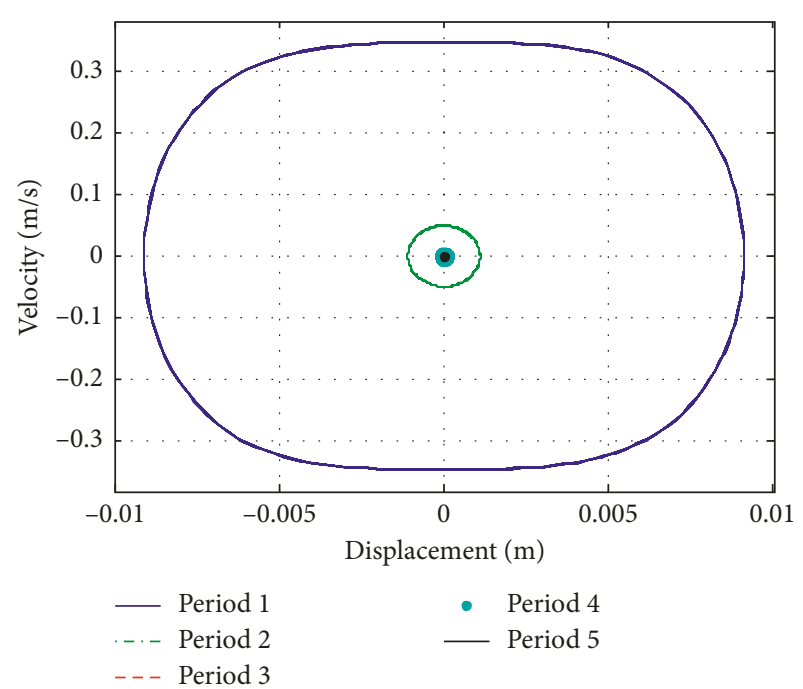

(b)

FIGURE 8: Five periodic shells' (a) displacement time history curves and (b) phase diagrams.

carried out under effective negative mass taking the external excitation frequency $\omega=7 \mathrm{~Hz}$. Figure 8 shows the vibration time history curves and phase diagrams of the shells in five periods. Figure 9 shows the oscillators in five periods.

From Figures 8 and 9, some time later, the vibration amplitude of each shell and oscillator no longer attenuates but has stabilized; the displacement-velocity phase diagrams of the shell and the oscillator both consist of a circle, and all the shells and oscillators of this periodic structure have achieved steady-state vibration.

Now, let us compare the shell vibration in each period. From period 1 through period 5 , the shell vibration gradually decreases. This vibration is almost zero in the last period. The internal Duffing oscillator shows very similar vibration characteristics to the shell. This indicates that vibration loaded on the shell in period 1 cannot be propagated in this periodic structure but is suppressed, which concurs with the characteristics of bandgap.

Figure 10 shows the vibration time histories of the shell and the Duffing oscillator in period 1 . Here, the dotted lines are the time histories of the shell, and the solid lines are the time histories of the Duffing oscillator. As can be observed, when the motion displacement of the shell $M$ is positive amplitude, the motion of the Duffing oscillator, $m$, is a negative amplitude; when the motion displacement of $M$ is a negative amplitude, the motion displacement of $m$ is also positive amplitude. This means that $M$ and $m$ always have an opposite motion displacement, and the vibrations of the shell and the oscillator are reverse phased. Here, the black curves are the time displacement curves of $m$ vibration of the 


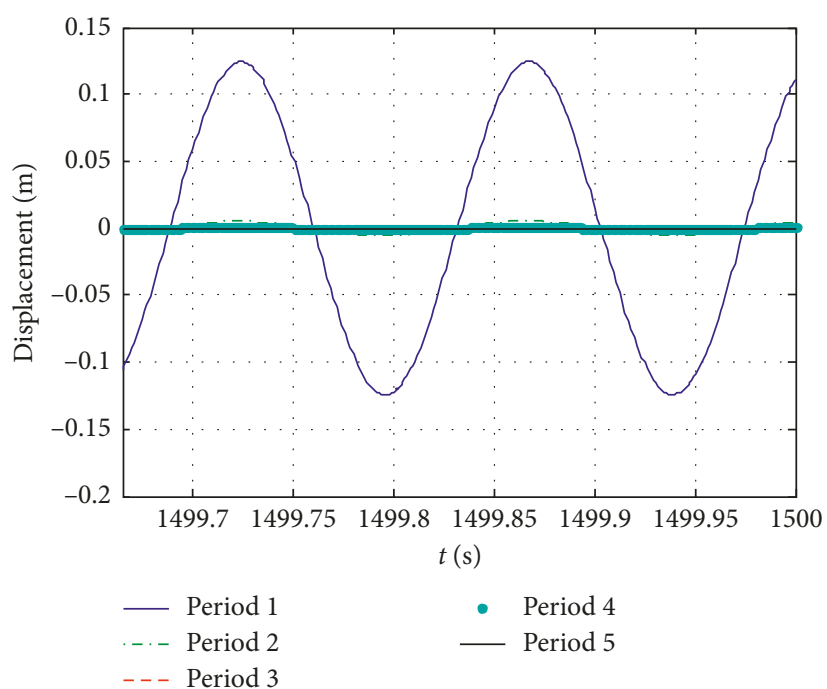

(a)

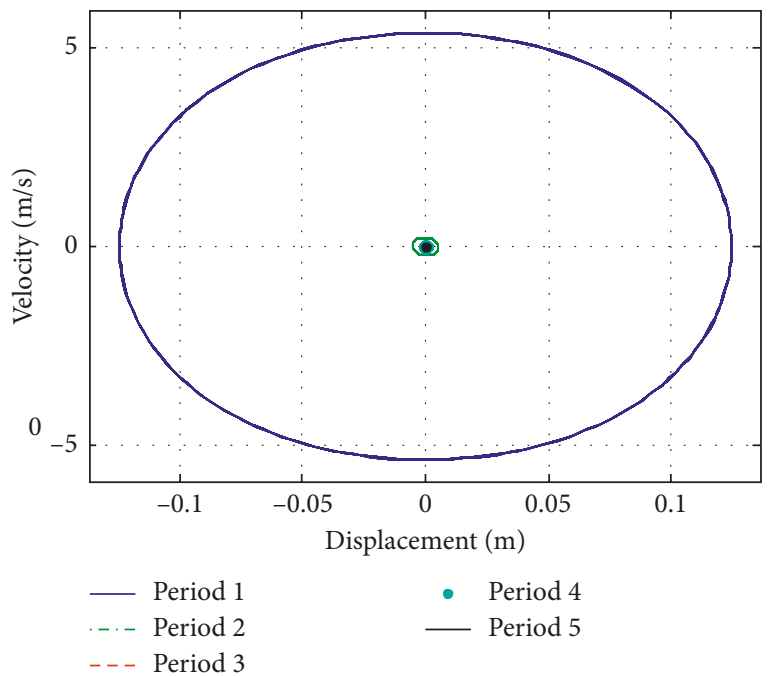

(b)

Figure 9: Five periodic oscillators' (a) displacement time history curves and (b) phase diagrams (effective negative mass).

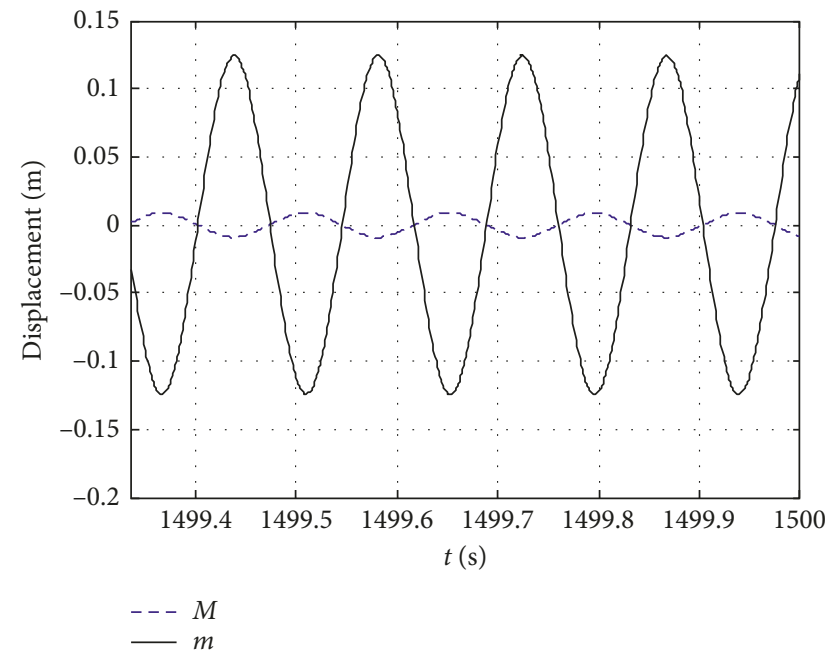

Figure 10: Displacement time history curves of shell and oscillator in period 1 .

internal mass bock. This is because $M$ has a larger mass than $m$, and under the same external excitation amplitude, objects with a smaller mass will find it easier to change their velocity, resulting in a larger displacement. Further analysis revealed that the Duffing oscillator has a greater momentum than the shell. Now, the system comprising the shell and the Duffing oscillator in individual periods has a negative momentum. As the effective velocity is positive, the system possesses negative mass, and consequently the system helps control vibration.

3.3.2. Effective Positive Mass Phenomenon in Bandgap. Based on the above analysis, bandgap in $6.35 \mathrm{~Hz}-7.67 \mathrm{~Hz}$, as shown in Figure 7, where the effective negative mass phenomenon occurs, meanwhile bandgap in $3.7 \mathrm{~Hz}-6.35 \mathrm{~Hz}$ should be the effective positive mass region. Analysis is carried out under effective positive mass taking the external excitation frequency $\omega=5 \mathrm{~Hz}$. Figure 11 shows the vibration time history curves and phase diagrams of the shells in five periods, and Figure 12 shows the oscillators in five periods.

From Figures 11 and 12, all the shells and oscillators of this periodic structure have achieved steady-state vibration. The vibrations loaded on the shell and oscillators in period 1 cannot be propagated in this periodic structure which concurs with the characteristics of bandgap.

Figure 13 shows the vibration time histories of the shell and the Duffing oscillator in period 1. As can be observed, when the motion displacement of the shell $M$ is positive amplitude, the motion of the Duffing oscillator, $m$, is also of the same positive amplitude. $M$ and $m$ always have the same motion displacement, and the vibrations of the shell and the oscillator in the same period are cophasal. Now, the system in every period must have a positive momentum, and the system helps control vibration just as effective as negative mass systems does. That is, effective positive mass phenomenon also occurs in the bandgap of nonlinear metamaterial.

3.3.3. Discussion on Effective Zero Mass. As analyzed above, when the effective mass gradually changes from negative to positive through a frequency point at which the effective mass is zero, the place corresponding to zero mass is where the bandgap cutoff frequency stands. Existence of nonlinear factor, however, makes a minor difference to the frequency point at zero mass, and it would be possible that the actual cutoff frequency of bandgap cannot be found. To solve this problem, we can closely sample the external excitation frequency so as to bind the bandgap cutoff frequency of the nonlinear system, i.e., the point of zero-mass frequency. 


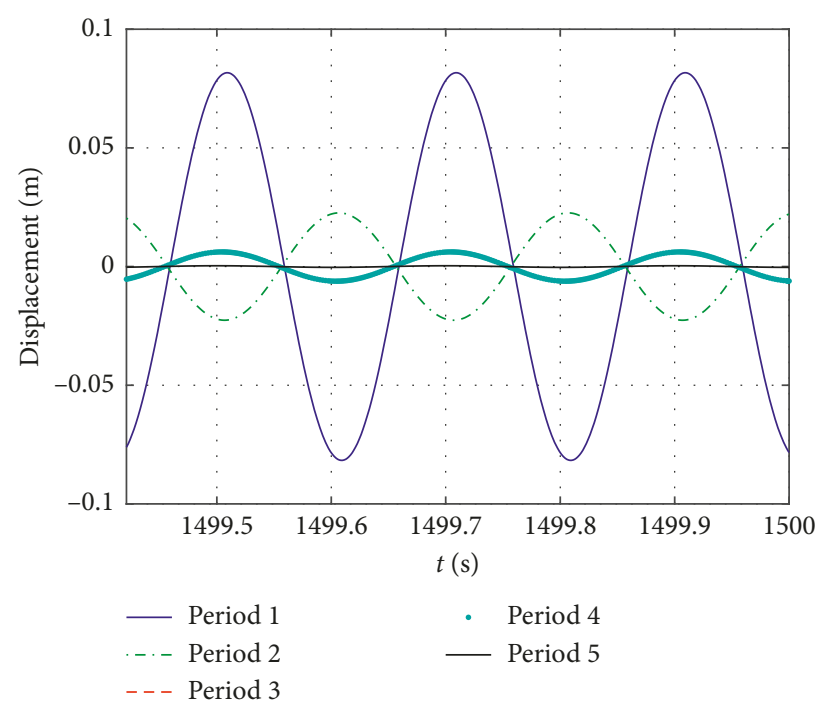

(a)

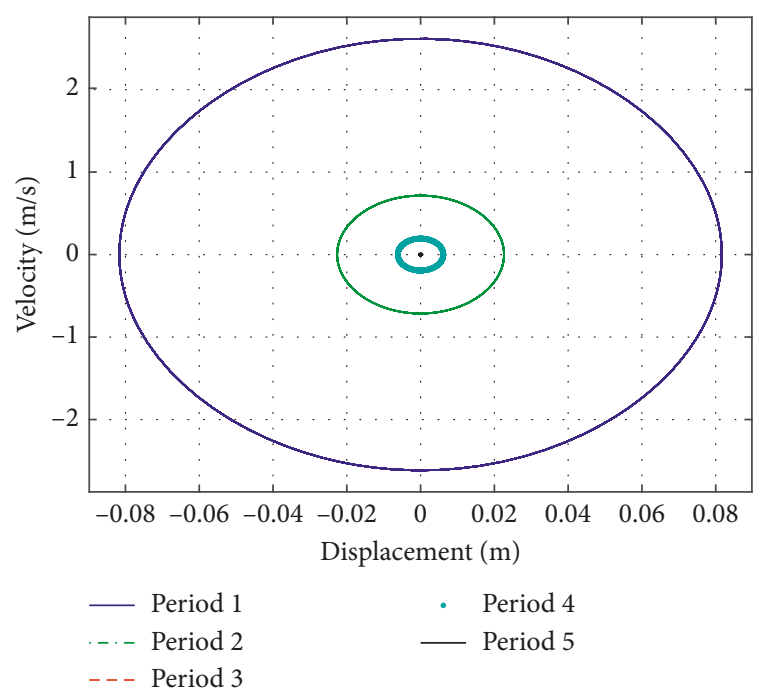

(b)

FIGURE 11: Five periodic shells' (a) displacement time history curves and (b) phase diagrams.

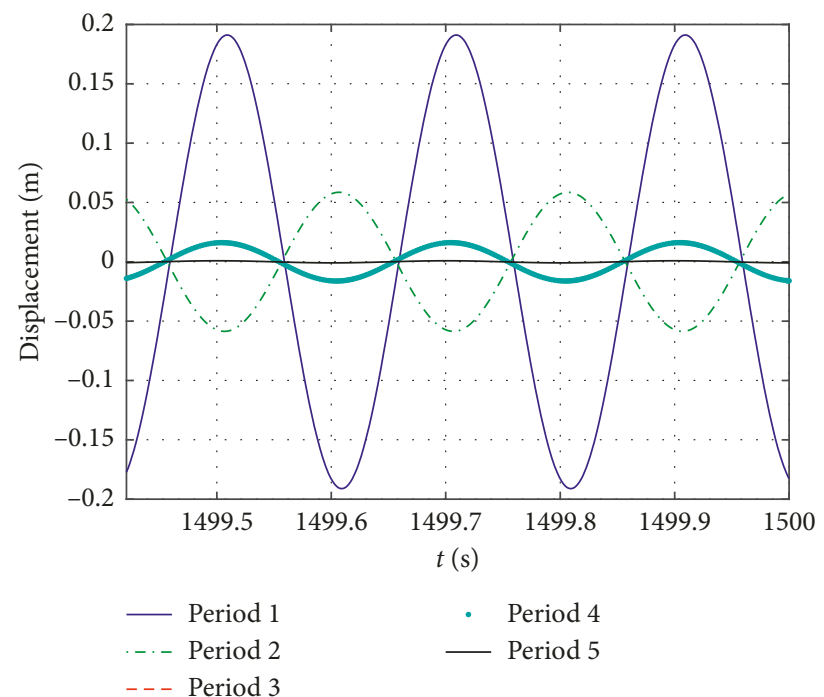

(a)

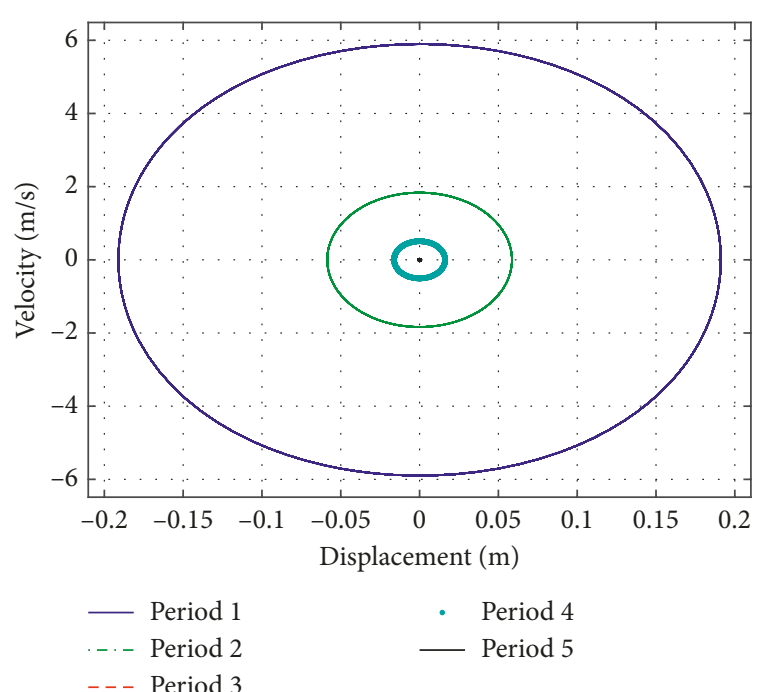

(b)

Figure 12: Five periodic oscillators' (a) displacement time history curves and (b) phase diagrams (effective positive mass).

Finally, with a constant nonlinear factor $n=10$, shell mass $\mathrm{M}=0.1011 \mathrm{~kg}$, internal oscillator mass $\mathrm{m}=0.04647 \mathrm{~kg}$, internal nonlinear spring linear rigidity $\mathrm{k}=37 \mathrm{~N} / \mathrm{m}$, and external linear spring damping coefficient $c=0.1 \mathrm{NS} / \mathrm{m}$, we found the frequency point at zero mass to be $8.2205 \mathrm{~Hz}$. Figures 14 and 15 show the time histories of the shells and the Duffing oscillators using this frequency point. From these curves, the shells and Duffing oscillators in all the periods are moving. From the phase diagrams, both the shells and the Duffing oscillators have reached a steady state, and the shells in all the periods have the same maximum displacements as the Duffing oscillators in all the periods.
From these charts in Figures 14 and 15, the shells of all periodic units move in the same phase and with the same displacement pattern, keeping the spring $K$ linking the periodic units from deformation. If we ignore the motion of the internal Duffing oscillators from outside, the entire system will appear to be a rigid rod. The entire system maintains an in-phase translation while all the internal Duffing oscillators move in the same phase but in the opposite direction to the shells, and there is no phase difference among the internal Duffing oscillators. This is because a zero mass unit signifies that its inertia force is zero, making it appear that the entire system is composed of massless rigid bodies. It can be proved 


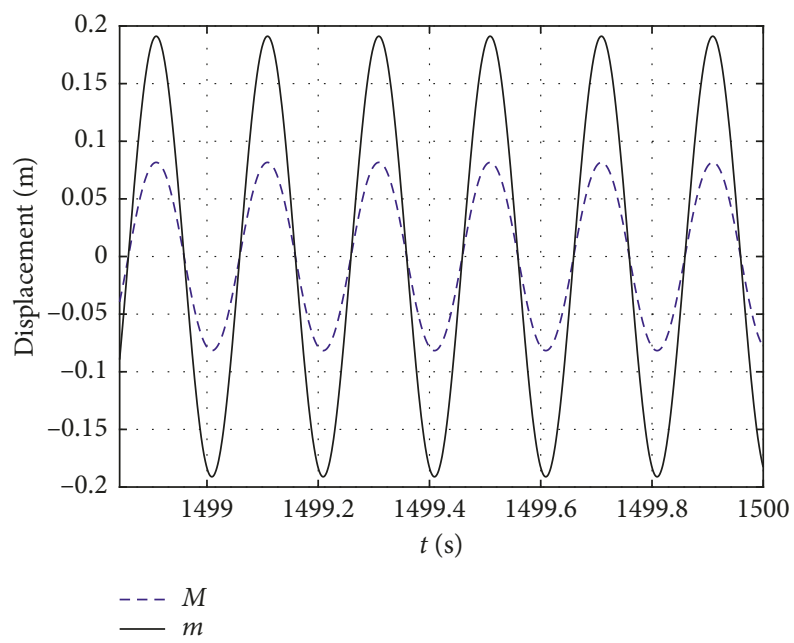

FIGURE 13: Displacement time history curves of shell and oscillator in period 1.

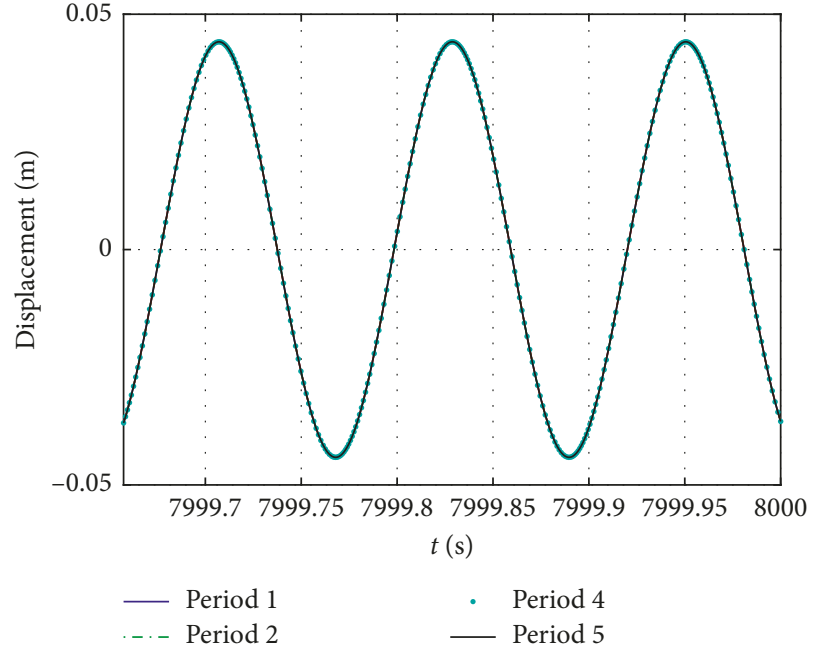

(a)

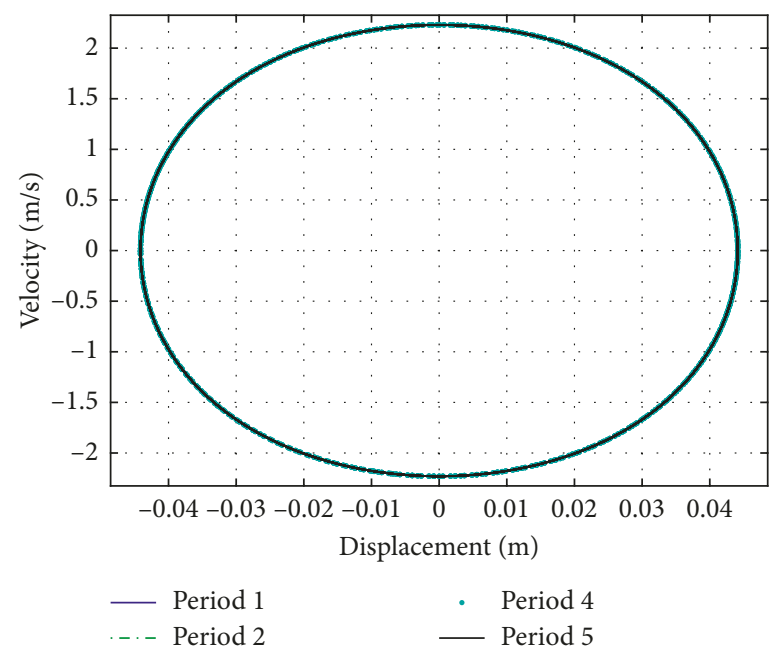

(b)

FIgURE 14: Five periodic shells' (a) displacement time history curves and (b) phase diagrams.

that the springs connecting the units will not deform, and the entire system looks as if one massless rigid rod is translating. The lattice kymatology of the periodic system can also provide an explanation. This phenomenon similar to zero mass is how electromagnetic waves propagate through a matching metamaterial with zero refraction index in electromagnetics. This material also features zero dielectric constant and zero magnetic permeability and no phase difference in the electromagnetic field.

3.4. Influence of the Excition Amplitude on the Start and Stop Frequencies of the Bandgap. As is known to all, for a linear system, the system response is irrelevant to the external excitation amplitude. For a nonlinear system, on the contrary, the system response is usually sensitive to and can vary with the external excitation amplitude. Hence, we are going to analyze how bandgap changes with the external excitation amplitude.

With a constant nonlinear factor $n$, we change external excitation amplitude to examine how it affects the periodic system again with shell mass $\mathrm{M}=0.1011 \mathrm{~kg}$, internal oscillator mass $m=0.04647 \mathrm{~kg}$, internal nonlinear spring linear rigidity $k=37 \mathrm{~N} / \mathrm{m}$, external linear spring damping coefficient $c=0.05 \mathrm{NS} / \mathrm{m}$, and rigid coefficient between springs connecting external large oscillators $K=117 \mathrm{~N} / \mathrm{m}$. If the nonlinear perturbation term is taken as $\varepsilon=0.01$, $n=1, f$ is taken as $1,2,3,5$, and 10 to calculate the vibration transmission characteristics. The result is presented in Figure 16. When the external excitation amplitude changes, 


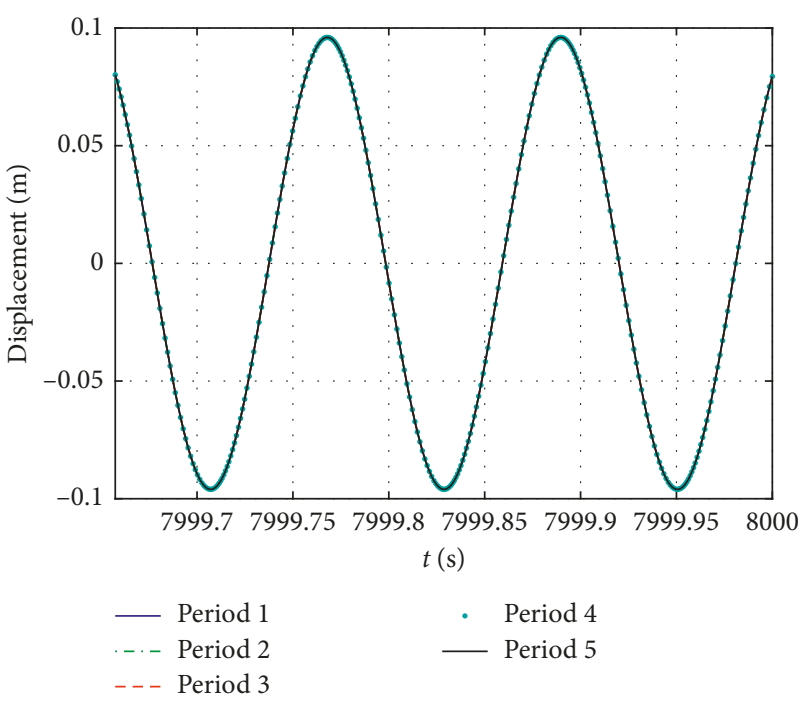

(a)

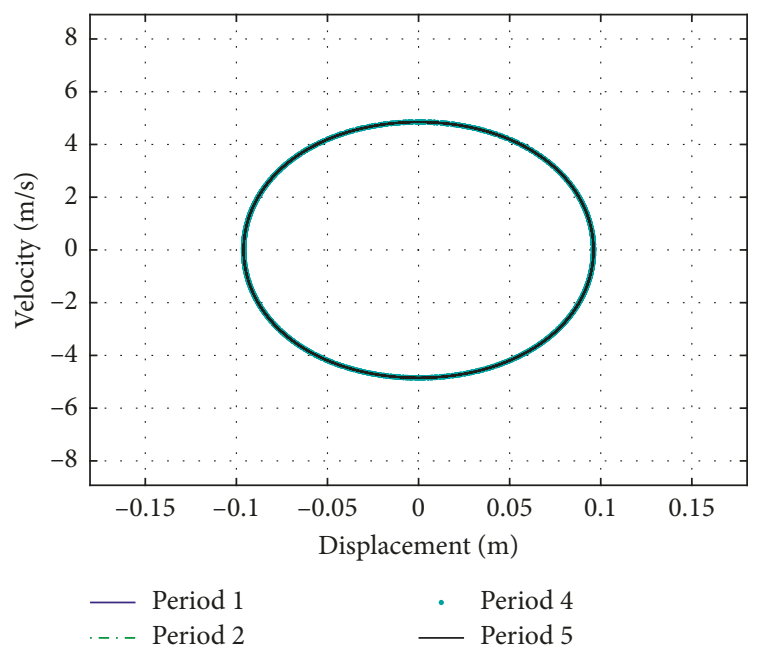

(b)

Figure 15: Five periodic oscillators' (a) displacement time history curves and (b) phase diagrams (effective zero mass).

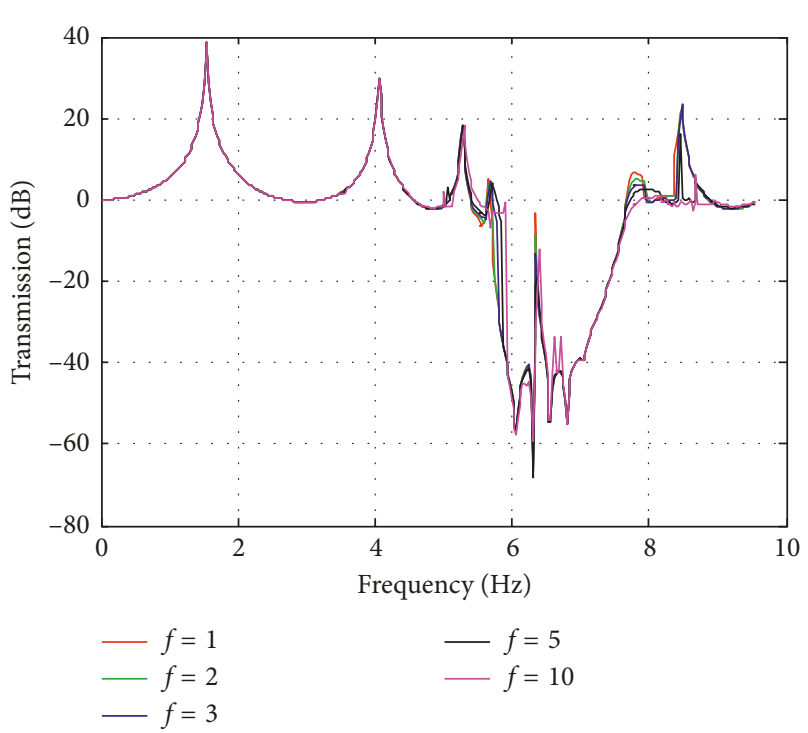

FIGURE 16: External excitation amplitude has changed the bandgap width.

so do the bandgap start and stop frequencies and the bandgap bandwidth. Figure 17 shows enlarged areas of the starting and cutoff frequencies.

As can be observed from Figure 17(a), the external excitation amplitude makes quite a big difference to the bandgap starting frequency. As the external excitation amplitude $f$ increases, the bandgap start frequency moves toward the high band; when $f$ decreases, the bandgap starting frequency moves toward the low band.

Figure 17(b) shows an enlarged view near the stop frequency. From this, we can see that, as $f$ increases, the bandgap stop frequency moves toward the high band; as $f$ decreases, the bandgap stop frequency moves toward the low band. As the transmission characteristic chart is much more separated than the previous two subsections, changing the $f$ value makes a greater difference to the starting and cutoff frequencies.

Through comparison above, we can see that external excitation amplitude makes a great difference to bandgap. As the external excitation amplitude increases, so does the bandgap starting frequency of the metamaterial. This will further reduce the bandgap width, which is negative for the application of vibration control. Hence, when a metamaterial of effective negative mass with Duffing oscillator is used for vibration control, the external excitation amplitude must be kept within a reasonable limit.

\section{Conclusions}

In this paper, the Lindstedt-Poincaré method is utilized to calculate the first-order perturbation solution of acoustic and optical branches for effective negative mass nonlinear acoustic metamaterial with pure Duffing oscillator. The starting and cutoff frequencies of bandgap are deduced. Then, the influence of mass ratio $\alpha$ and stiffness ratio $\beta$ and nonlinearity influence on starting and cutoff frequency of bandgap are discussed. The main conclusions can be drawn as follows:

(1) The nonlinear part of the dispersion curves solution has relationships with coefficient of nonlinear item, steady-state amplitude of Duffing oscillator, and $\beta$. Both soft and hard Duffing oscillators have effect on the starting and cutoff frequencies. The soft/hard characteristic of Duffing oscillator leads to the trend that both starting and cutoff frequencies move down/up relative to the degraded linear system, and 


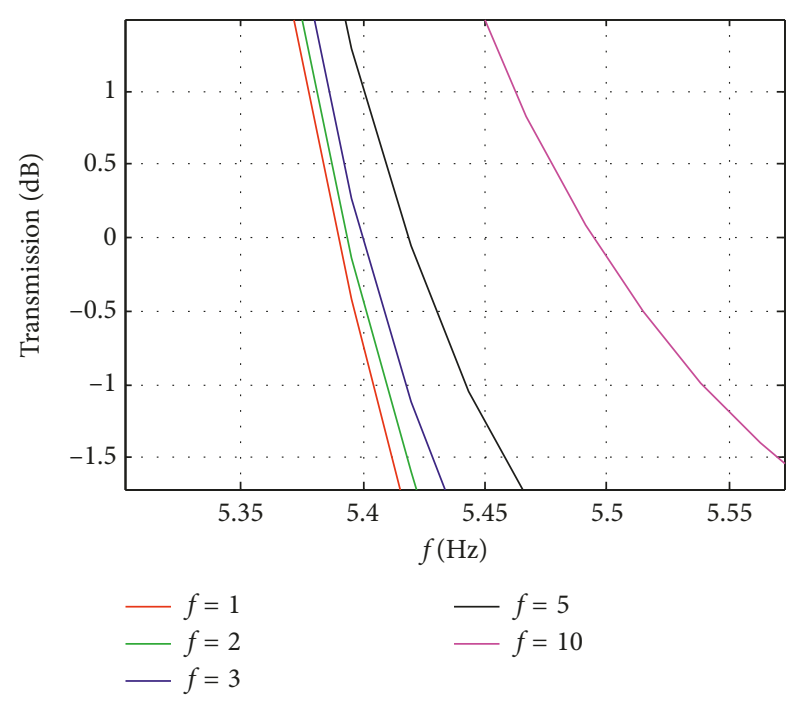

(a)

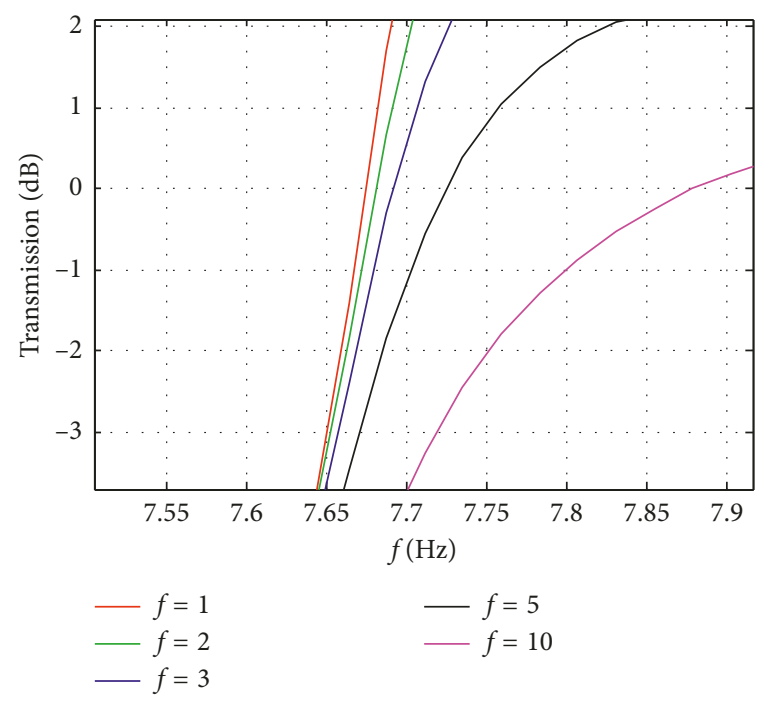

(b)

FIgURE 17: External excitation amplitude has changed the starting and cutoff frequencies. (a) Starting frequency region. (b) Cutoff frequency region.

the stronger the nonlinearity is the more obvious the trend is.

(2) Both starting and cutoff frequencies of this nonlinear metamaterial bandgap depend on $\alpha$ and $\beta$. The starting frequency is lower than the resonant frequency, and it is monotonically increasing with $\alpha$ and decreasing with $\beta$. The cutoff frequency is only decreasing with mass ratio.

(3) The actual nonlinear metamaterial bandgap region is wider than effective negative mass region. The frequency range from the resonant frequency to cutoff frequency is corresponding to effective negative mass. The frequency range from starting frequency to the resonant frequency is corresponding to effective positive mass. The vibration attenuation of both effective negative and effective positive mass occurs in bandgap.

(4) Effective zero-mass phenomenon also exists in this nonlinear metamaterial. This is corresponding to the cutoff frequency. On this frequency, there is no relative motion for every shell of this nonlinear metamaterial, and every Duffing oscillator also has the same characteristics. The phases of shell and Duffing oscillator in every period are opposite, and the aggregate momentum of every periodic unit is close to zero.

(5) The external excitation amplitude has influence on bandgap. As the amplitude increases, the starting and cutoff frequencies shift to the high-frequency band.

\section{Data Availability}

The data used to support the findings of this study are available from the corresponding author upon request.

\section{Disclosure}

Gao Ming now works in College of Mechanical and Electronic Engineering, Shandong Agricultural University in Taian, China.

\section{Conflicts of Interest}

The authors declare that they have no conflicts of interest.

\section{Acknowledgments}

The authors acknowledge the State Key Research Development Program of China (Grant no. 2016YFC0600708), the National Natural Science Foundation of China (Grant nos. 11172198 and 51605264), the National Basic Research Program of China (Grant nos. 2013CB035402 and 2014CB046800), the Shandong Province Higher Educational Science and Technology Program (Grant no. J15LH04), the Natural Science Foundation of Shandong Province (Grant nos. ZR2018 and MEE001), and the State Key Laboratory of Open Funds (Grant no. MDPC201601).

\section{References}

[1] S. A. Cummer, J. Christensen, and A. Alù, "Controlling sound with acoustic metamaterials," Nature Reviews Materials, vol. 1, no. 3, p. 16001, 2016.

[2] A. Nanda and M. A. Karami, "Tunable bandgaps in a deployable metamaterial," Journal of Sound and Vibration, vol. 424, pp. 120-136, 2018.

[3] F. Wu, "Achieving highly efficient and broad-angle polarization beam filtering using epsilon-near-zero metamaterials mimicked by metal-dielectric multilayers," Journal of Applied Physics, vol. 123, no. 9, article 093103, 2018.

[4] L. Xi, H. Zhai, and L. Li, "A compact low profile dualpolarized filtering antenna with metamaterial for wideband base station applications," Microwave and Optical Technology Letters, vol. 60, no. 1, pp. 64-69, 2018.

[5] Z. Xu, J. Tong, and F. Wu, "Magnetorheological elastomer vibration isolation of tunable three-dimensional locally 
resonant acoustic metamaterial," Solid State Communications, vol. 271, pp. 51-55, 2018.

[6] Y. Liao, Y. Chen, G. Huang, and X. Zhou, "Broadband lowfrequency sound isolation by lightweight adaptive metamaterials," Journal of Applied Physics, vol. 123, no. 9, article 091705, 2018.

[7] T. Jiang and Q. He, "Dual-directionally tunable metamaterial for low-frequency vibration isolation," Applied Physics Letters, vol. 110, no. 2, article 021907, 2017.

[8] P. Marinova, S. Lippert, and O. von Estorff, "On the numerical investigation of sound transmission through double-walled structures with membrane-type acoustic metamaterials," Journal of the Acoustical Society of America, vol. 142, no. 4, pp. 2400-2406, 2017.

[9] M. Oudich, X. Zhou, and M. Badreddine Assouar, "General analytical approach for sound transmission loss analysis through a thick metamaterial plate," Journal of Applied Physics, vol. 116, no. 19, article 193509, 2014.

[10] Z. Cheng and Z. Shi, "Novel composite periodic structures with attenuation zones," Engineering Structures, vol. 56, pp. 1271-1282, 2013.

[11] X. Liu, Z. Shi, Y. L. Mo, and Z. Cheng, "Effect of initial stress on attenuation zones of layered periodic foundations," Engineering Structures, vol. 121, pp. 75-84, 2016.

[12] H. J. Xiang, Z. F. Shi, S. J. Wang, and Y. L. Mo, "Periodic materials-based vibration attenuation in layered foundations: experimental validation," Smart Materials and Structures, vol. 21, no. 11, article 112003, 2012.

[13] J. Huang and Z. Shi, "Application of periodic theory to rows of piles for horizontal vibration attenuation," International Journal of Geomechanics, vol. 13, no. 2, pp. 132-142, 2013.

[14] Y. Q. Yan, Z. B. Cheng, F.-Y. Menq, Y. L. Mo, Y. Tang, and Z. F. Shi, "Three dimensional periodic foundations for base seismic isolation," Smart Materials and Structures, vol. 24, no. 7, article 075006, 2015.

[15] S. Brûlé, E. H. Javelaud, S. Enoch, and S. Guenneau, "Experiments on seismic metamaterials: molding surface waves," Physical Review Letters, vol. 112, no. 13, article 133901, 2014.

[16] A. Colombi, P. Roux, S. Guenneau, P. Gueguen, and R. V. Craster, "Forests as a natural seismic metamaterial: Rayleigh wave bandgaps induced by local resonances," Scientific Reports, vol. 6, no. 1, article 19238, 2016.

[17] V. K. Dertimanis, L. A. Antoniadis, and E. N. Chatzi, "Feasibility analysis on the attenuation of strong ground motions using finite periodic lattices of mass-in-mass barriers," Journal of Engineering Mechanics-ASCE, vol. 142, no. 9, article 04016060, 2016.

[18] G. W. 't Hooft, "Comment on "negative refraction makes a perfect lens"," Physical Review Letters, vol. 87, no. 24, article 249701, 2001.

[19] J. Valentine, S. Zhang, T. Zentgraf et al., “Three-dimensional optical metamaterial with a negative refractive index," Nature, vol. 455, no. 7211, pp. 376-379, 2008.

[20] Z. Y. Liu, X. X. Zhang, Y. W. Mao et al., "Locally resonant sonic materials," Science, vol. 289, no. 5485, pp. 1734-1736, 2000.

[21] Z. Y. Liu, C. T. Chan, and P. Sheng, "Analytic model of phononic crystals with local resonances," Physical Review B, vol. 71, no. 1, article 014103, 2005.

[22] G. W. Milton and J. R. Willis, "On modifications of Newton's second law and linear continuum elastodynamics," Proceedings of the Royal Society A, vol. 463, no. 2079, pp. 855-880, 2007.
[23] J. R. Willis, "Exact effective relations for dynamics of a laminated body," Mechanics of Materials, vol. 41, no. 4, pp. 385-393, 2009.

[24] H. H. Huang and C. T. Sun, "Wave attenuation mechanism in an acoustic metamaterial with negative effective mass density," New Journal of Physics, vol. 11, no. 1, article 013003, 2009.

[25] H. H. Huang, C. T. Sun, and G. L. Huang, "On the negative effective mass density in acoustic metamaterials," International Journal of Engineering Science, vol. 47, no. 4, pp. 610-617, 2009.

[26] S. S. Yao, X. M. Zhou, and G. K. Hu, "Experimental study on negative effective mass in a $1 \mathrm{D}$ mass-spring system," New Journal of Physics, vol. 10, no. 10, article 043020, 2008.

[27] S. H. Lee, C. M. Park, Y. M. Seo, Z. G. Wang, and C. K. Kim, "Acoustic metamaterial with negative density," Physics Letters A, vol. 373, no. 48, pp. 4464-4469, 2009.

[28] K. T. Tan, H. H. Huang, and C. T. Sun, "Optimizing the bandgap of effective mass negativity in acoustic metamaterials," Applied Physics Letters, vol. 101, no. 24, article 241902, 2012

[29] A. O. Krushynska, V. G. Kouznetsova, and M. G. D. Geers, "Towards optimal design of locally resonant acoustic metamaterials," Journal of Mechanics and Physics of Solids, vol. 71, pp. 179-196, 2014.

[30] H. J. Chen, S. L. Zhai, C. L. Ding, S. Liu, C. R. Luo, and X. P. Zhao, "Meta-atom cluster acoustic metamaterial with broadband negative effective mass density," Journal of Applied Physics, vol. 115, no. 15, article 054905, 2014.

[31] X. Wang, Z.-J. Wen, Y. Jiang, and H. Huang, "Experimental study on mechanical and acoustic emission characteristics of rock-like material under non-uniformly distributed loads," Rock Mechanics and Rock Engineering, vol. 51, no. 3, pp. 729-745, 2018.

[32] J. J. Park, C. M. Park, K. J. B. Lee, and S. H. Lee, "Acoustic superlens using membrane-based metamaterials," Applied Physics Letters, vol. 106, no. 5, article 051901, 2015.

[33] A. F. Vakakis, M. E. King, and A. J. Pearlstein, "Forced localization in a periodic chain of non-linear oscillators," International Journal of Non-Linear Mechanics, vol. 29, no. 3, pp. 429-447, 1994.

[34] A. F. Vakakis and M. E. King, "Nonlinear wave transmission in a monocoupled elastic periodic system," Journal of the Acoustical Society of America, vol. 98, no. 3, pp. 1534-1546, 1995.

[35] G. Chakraborty and A. K. Mallik, "Dynamics of a weekly nonlinear periodic chain," International Journal of Non-Linear Mechanics, vol. 36, no. 2, pp. 375-389, 2001.

[36] B. S. Lazarov and J. S. Jensen, "Low-frequency bandgaps in chains with attached non-linear oscillators," International Journal of Non-Linear Mechanics, vol. 42, no. 10, pp. 11861193, 2007.

[37] A. Marathe and A. Chatterjee, "Wave attenuation in nonlinear periodic structures using harmonic balance and multiple scales," Journal of Sound and Vibration, vol. 289, no. 4-5, pp. 871-888, 2006.

[38] R. K. Narisetti, M. J. Leamy, and M. Ruzzene, “A perturbation approach for predicting wave propagation in onedimensional nonlinear periodic structures," Journal of $\mathrm{Vi}$ bration and Acoustics of the ASME, vol. 132, no. 3, article 031001, 2010.

[39] M. I. Hussein, M. J. Leamy, and M. Ruzzene, "Dynamics of phononic materials and structures: historical origins, recent 
progress, and future outlook," Applied Mechanics Reviews, vol. 66, no. 4, article 040802, 2014.

[40] X. Wen, J. Wen, D. Yu et al., Phononic Crystals, National Defense Industry Press, Beijing, China, 2009. 


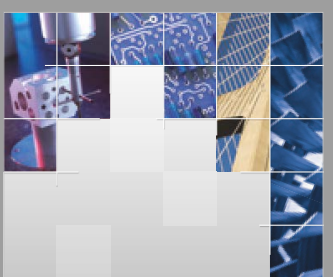

\section{Enfincering}
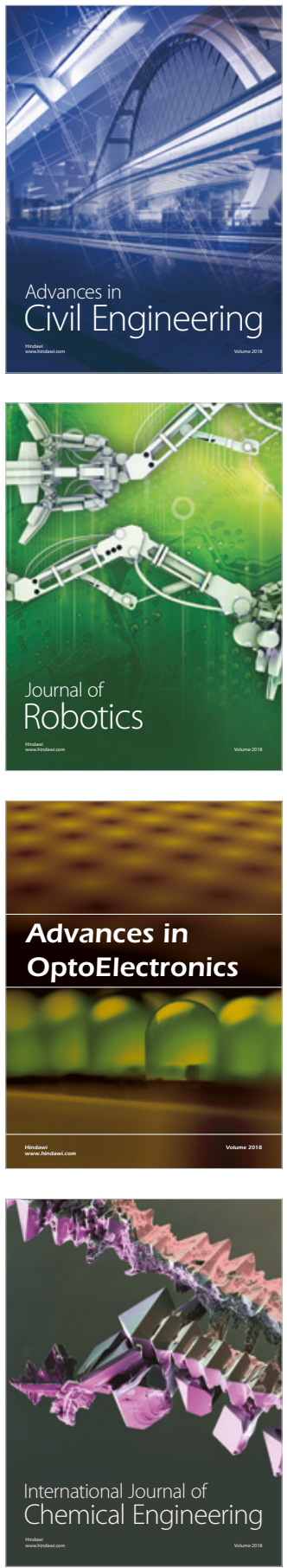

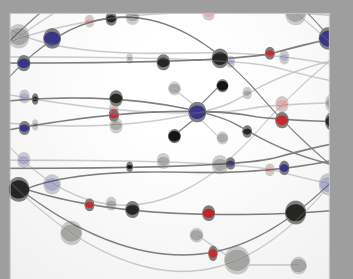

\section{Rotating \\ Machinery}

The Scientific World Journal

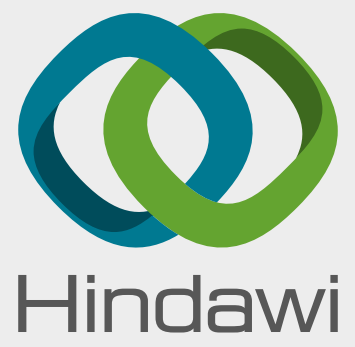

Submit your manuscripts at

www.hindawi.com
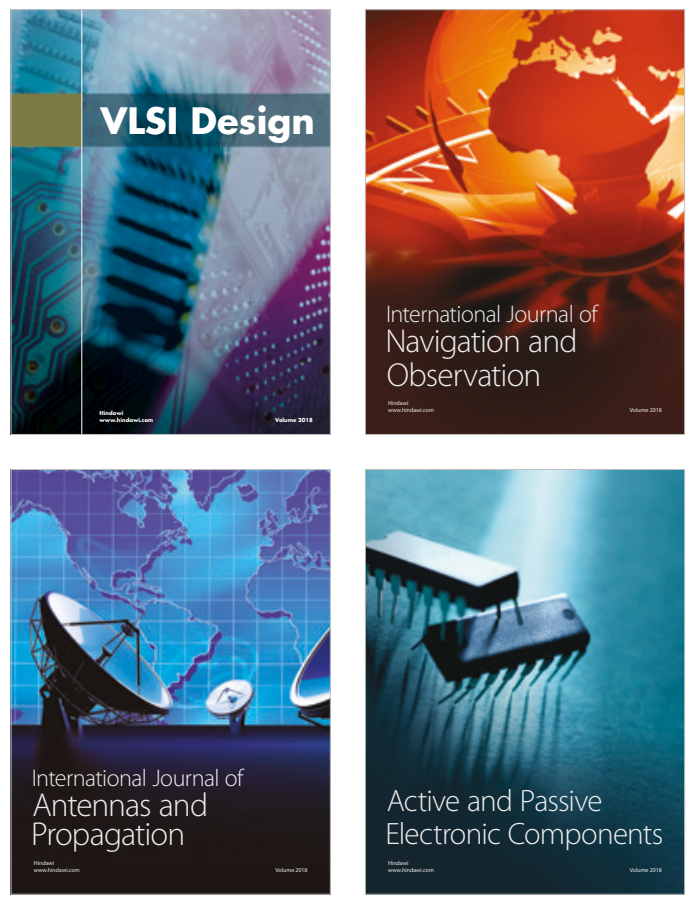
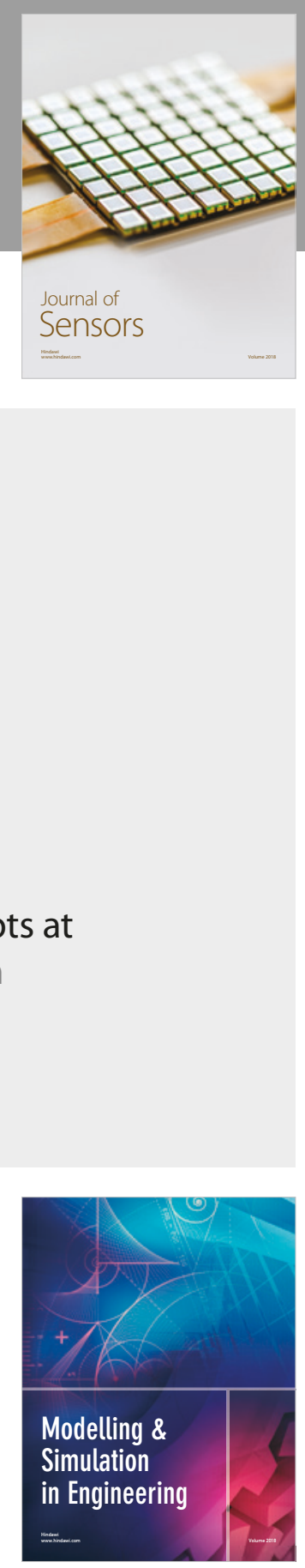

\section{Advances \\ Multimedia}
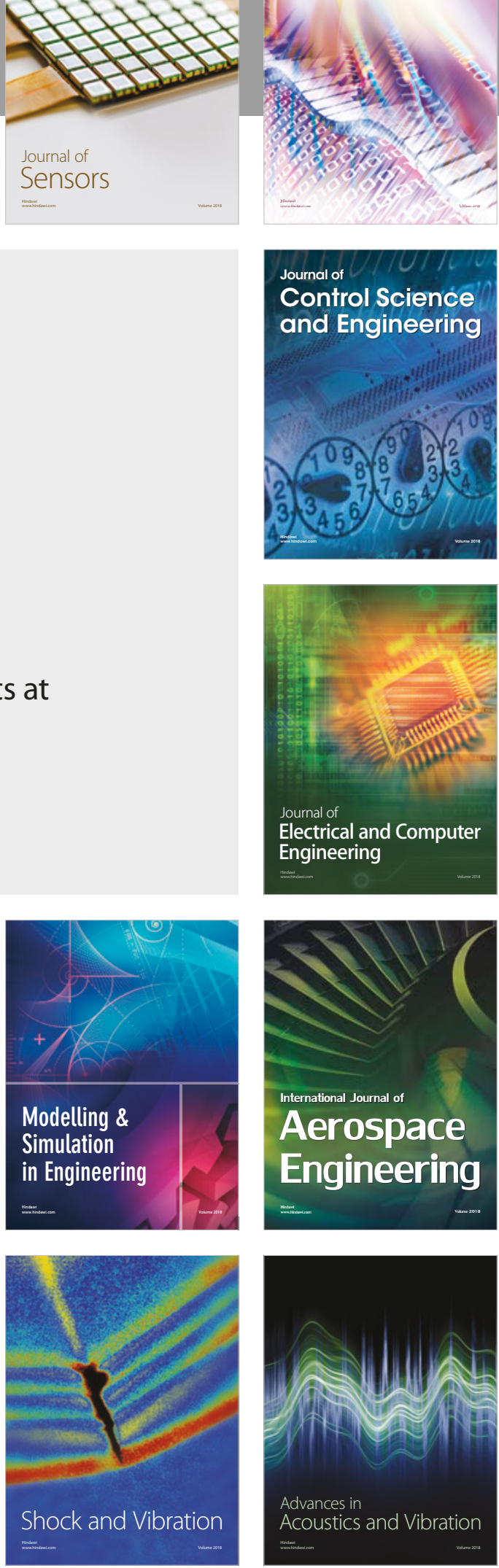